\title{
Relay fusion frames for Hilbert spaces
}

Guoqing Hong ${ }^{1 *}$ and Pengtong $\mathrm{Li}^{1}$

"Correspondence:

guoqinghong@nuaa.edu.cn

${ }^{1}$ Department of Mathematics,

Nanjing University of Aeronautics

and Astronautics, Nanjing, P.R. China

\begin{abstract}
A new notion of frames, called the relay fusion frames, for Hilbert spaces has been introduced by the authors. It provides a mathematical framework for applications that require the transmission of signals over long distances or need to expand the coverage of wireless networks. The technique described in this paper is not only a natural technique suitable for the applications of relay communication systems, but also can be regarded as a natural generalization of fusion frames or even g-frames. We transfer some common properties in general frames and fusion frames to relay fusion frames with the definition of the relay fusion frames and their operators. In particular, besides canonical duality, we obtain two new dualities of the relay fusion frames. Moreover, we prove that relay fusion frames are stable under small perturbations.
\end{abstract}

MSC: Primary 42C15; secondary 46C99; 41A58

Keywords: Frame; Fusion frame; Relay fusion frame; Dual frame; Stability

\section{Introduction}

The notion of frames for Hilbert spaces was first introduced by Duffin and Shaeffer [9] in 1952 for investigating some deep problems in non-harmonics Fourier series, and popularized from then on. Frames were developed as a powerful tool in signal processing, coding theory, communication theory, sampling theory and many other fields.

Fusion frames (frame of subspaces) arose naturally as a generalization of frames. They are collections of closed subspaces and weights that allow the reconstruction of each element of a Hilbert space from packets of coefficients. Fusion frames are very useful in areas such as sensor networks, neurology, distributing sensing, parallel processing and packet encoding, among others.

\subsection{Background of research}

In the study of application aspects of frame and fusion frame theory one of the most important concepts is the signal processing. However, the conditions to transmission signals in "classical frame theory" are very restrictive-the long distance between transmitters and receivers is possible. This makes it hard to transmit signals and this is the reason that one might look for a more flexible tool.

In applications, an input signal is converted into the data vectors. In an ideal setting, these data vectors can be transmitted directly to the data receiver and the signal can be reconstructed by the receiver using frame and dual frame elements. However, in real implementations, sometimes the data vectors cannot be received directly by the receiver, such as relay communication [12, 20], distributed sensor relay system [23], cooperative

(c) The Author(s) 2019. This article is distributed under the terms of the Creative Commons Attribution 4.0 International License (http://creativecommons.org/licenses/by/4.0/), which permits unrestricted use, distribution, and reproduction in any medium, provided you give appropriate credit to the original author(s) and the source, provide a link to the Creative Commons license, and indicate if changes were made. 
communication [18], satellite communication [17], microwave communication [16], and ultrashort wave communication [15].

When using microwave and ultrashort wave to transmit signals, if the distance between two terminals exceeds the visual distance, the signals attenuate quickly and the quality cannot be guaranteed. If a number of relay stations are set up between two terminals, the relay stations will transmit the signals from the previous station after amplification, shaping and carrier frequency conversion to the next station, which can extend the communication distance and maintain good communication quality, and at the same time help small power equipment to expand the signals, providing a new network mode. This mode can realize the amplification and continuation of network signals and solve the problem of distance for network construction.

In virtue of the role of relay stations, microwave communication transmits signals hundreds or even thousands of kilometers away. They can be used to improve services in part of the main coverage area where poor signals are received due to geographical constraints. The receiving and transmitting radius of the relay stations covers a large area.

A company hopes to connect all the networks of its nearby production plants, workshops, management centers, so as to facilitate resource sharing, unified management and maximize the use of information. In university campuses, independent intranets in teaching buildings, student dormitories and computing centers also need to be set up together to facilitate the access of students and teachers to campus networks, and so on. When the local area networks need to be connected are too far apart and the signals are weak, we need to use wireless relay technology to connect and build the network to achieve wireless bridging between the local area networks.

Under these circumstances, the applications cannot be modeled naturally by the traditional frame systems. In order to deal with these applications of frame new methods have to be developed. A relay fusion frame theory with local frame system is therefore in demand.

Relay fusion frames are such tools. A relay fusion frame for a vector space equipped with an inner product can also process signals, but the distance between transmitters and receivers is not required. In this paper, we present relay fusion frames theory in Hilbert spaces.

Relay fusion frame system is a device that can receive and transmit signals over a long distance. It provides a mathematical model for applications requiring long distance transmission of signals. From this point of view, the traditional frame theory can be regarded as a system with "real-time communication" function without involving the relay technique, while the relay fusion frame theory can be thought of as a system with "relay communication" function.

The advantage of the relay fusion frame system is that it utilizes relay technique to relay signals from one relay point to the next relay point, and forming a new network coverage area, thus constituting multiple relay coverage modes, and ultimately achieving the purpose of extending the coverage of the network. The relay mode networking method is extremely versatile. Nowadays, the network has begun to be widely used. In many places, because the site is large or has obstacles, the network coverage of the wireless device does not reach the distance we need or is hindered in the middle. At this time, if we use the relay mode to connect to the wireless network, we can meet the networking requirements. 


\subsection{Related approaches}

In view of the relationships between fusion frames and distributed processing proposed by Cazassa, Kutyniok and Li in the fundamental paper [5], we can also establish the connection between the relay fusion frames and the distributed sensor relay systems and the cooperative communications in a similar way. But in our approaches, we hope to adopt a more intuitive way to introduce the relay fusion frame based on frame and fusion frame theory. We remark that besides applications, relay fusion frames for Hilbert spaces or Banach spaces are interesting and natural objects to study from both pure and applied mathematics of the theory point of view, so our work is not trivial.

\subsection{Contents}

The paper is organized as follows. In Sect. 2 we recall frames, fusion frames and their operators. In Sect. 3 we introduce the definition of relay fusion frames. We will show that a relay fusion frame behaves as a generalization of fusion frames or even g-frames, which also provide an associated analysis and synthesis operator, a frame operator and a dual object. Particularly, in addition to canonical dual, we give two new dualities of the relay fusion frames. We end this section with the Q-dual relay fusion frames of relay fusion frames. In Sect. 4 we study the relay fusion frame systems and we also get some useful results about it. Finally, in Sect. 5 we prove that relay fusion frames are stable under small perturbations.

\section{Preliminaries}

In this section we briefly recall the definitions of frames and fusion frames and their operators. For more information about the theory and applications of frames and fusion frames we refer to $[2,6,11]$ and $[1,4,10,19]$, respectively. First, let us introduce some notations.

\subsection{Notation}

Let $H$ and $K$ be separable complex Hilbert spaces and let $\mathcal{B}(H, K)$ be the space of all the bounded linear operators from $H$ to $K$ (if $H=K$ we write $\mathcal{B}(H)$ ). $\mathbb{I}, \mathbb{J}_{i}$ and every $\mathbb{K}_{i j}$ will denote generic countable (or finite) index sets. We use $I_{H}$ to denote the identity operator on $H$.

If $W \subseteq H$ and $V \subseteq K$ are subspaces, then we let $\pi_{W} \in \mathcal{B}(H)$ and $\tau_{V} \in \mathcal{B}(K)$ denote the orthogonal projections onto the subspaces $W$ and $V$, respectively.

\subsection{Frames}

Definition 1 A sequence $\mathcal{F}=\left\{f_{i}\right\}_{i \in \mathbb{I}}$ of elements in $\mathrm{H}$ is said to be a frame for $\mathrm{H}$ if there exist constants $\alpha, \beta>0$ such that

$$
\alpha\|f\|^{2} \leq \sum_{i \in \mathbb{I}}\left|\left\langle f, f_{i}\right\rangle\right|^{2} \leq \beta\|f\|^{2}, \quad \forall f \in H .
$$

The numbers $\alpha, \beta$ are called frame bounds. A frame $\mathcal{F}$ is tight if we can choose $\alpha=\beta$ as frame bounds. Provided (1) holds with $\alpha=\beta=1$, we call $\mathcal{F}$ a Parseval frame.

Definition 2 Let $\mathcal{F}$ be a frame for $\mathrm{H}$.

(i) The frame transform or analysis operator of $\mathcal{F}$ is

$$
T_{\mathcal{F}}: H \mapsto \ell^{2}(\mathbb{I}), \quad T_{\mathcal{F}}(f)=\left\{\left\langle f, f_{i}\right\rangle\right\}_{i \in \mathbb{I}}
$$


(ii) The adjoint of $T_{\mathcal{F}}$ is given by

$$
T_{\mathcal{F}}^{*}: \ell^{2}(\mathbb{I}) \mapsto H, \quad T_{\mathcal{F}}^{*}\left(\left\{c_{i}\right\}_{i \in \mathbb{I}}\right)=\sum_{i \in \mathbb{I}} c_{i} f_{i} .
$$

$T_{\mathcal{F}}^{*}$ is called the pre-frame operator or the synthesis operator of $\mathcal{F}$.

(iii) The frame operator of $\mathcal{F}$ is

$$
S_{\mathcal{F}}=T_{\mathcal{F}}^{*} T_{\mathcal{F}}: H \mapsto H, \quad S_{\mathcal{F}}(f)=\sum_{i \in \mathbb{I}}\left\langle f, f_{i}\right\rangle f_{i} .
$$

Definition 3 Let $\mathcal{F}=\left\{f_{i}\right\}_{i \in \mathbb{I}}$ and $\widetilde{\mathcal{F}}=\left\{\widetilde{f}_{i}\right\}_{i \in \mathbb{I}}$ be frames for $\mathrm{H}$. Then $\widetilde{\mathcal{F}}$ is said to be a dual frame of $\mathcal{F}$ if the following reconstruction formula holds:

$$
f=\sum_{i \in \mathbb{I}}\left\langle f, f_{i}\right\rangle \widetilde{f}_{i}, \quad \forall f \in H
$$

or, equivalently,

$$
T_{\widetilde{\mathcal{F}}}^{*} T_{\mathcal{F}}=I_{H}
$$

We call $\left\{S_{\mathcal{F}}^{-1} f_{i}\right\}_{i \in \mathbb{I}}$ the canonical dual frame of $\mathcal{F}$.

\subsection{Fusion frames}

Definition 4 Let $\left\{W_{i}\right\}_{i \in \mathbb{I}}$ be a sequence of closed subspaces of $\mathrm{H}$, and let $\left\{v_{i}\right\}_{i \in \mathbb{I}} \in l^{\infty}(\mathbb{I})$ such that $v_{i}>0$ for every $i \in \mathbb{I}$. The sequence $\left\{\left(W_{i}, v_{i}\right)\right\}_{i \in \mathbb{I}}$ is said to be a fusion frame for $H$ if there exist numbers $0<\alpha \leq \beta<\infty$ such that

$$
\alpha\|f\|^{2} \leq \sum_{i \in \mathbb{I}} v_{i}^{2}\left\|\pi_{W_{i}}(f)\right\|^{2} \leq \beta\|f\|^{2}, \quad \forall f \in H .
$$

We call $\alpha$ and $\beta$ the fusion frame bounds. A fusion frame $\left\{\left(W_{i}, v_{i}\right)\right\}_{i \in \mathbb{I}}$ is called a tight fusion frame if the constants $\alpha$ and $\beta$ can be chosen so that $\alpha=\beta$. If $\alpha=\beta=1$ we say that it is a Parseval fusion frame.

Definition 5 Let $\left\{\left(W_{i}, v_{i}\right)\right\}_{i \in \mathbb{I}}$ be a fusion frame for $\mathrm{H}$ and let

$$
\left(\sum_{i \in \mathbb{I}} \oplus W_{i}\right)_{\ell^{2}}=\left\{\left\{f_{i}\right\}_{i \in \mathbb{I}} \mid f_{i} \in W_{i}(\forall i \in \mathbb{I}), \sum_{i \in \mathbb{I}}\left\|f_{i}\right\|^{2}<\infty\right\} .
$$

(i) The analysis operator of $\left\{\left(W_{i}, v_{i}\right)\right\}_{i \in \mathbb{I}}$ is defined by

$$
T_{\mathcal{W}}: H \mapsto\left(\sum_{i \in \mathbb{I}} \oplus W_{i}\right)_{\ell^{2}} \quad \text { with } T_{\mathcal{W}}(f)=\left\{v_{i} \pi_{W_{i}}(f)\right\}_{i \in \mathbb{I}}
$$

(ii) The synthesis operator of $\left\{\left(W_{i}, v_{i}\right)\right\}_{i \in \mathbb{I}}$ is defined by

$$
T_{\mathcal{W}}^{*}:\left(\sum_{i \in \mathbb{I}} \oplus W_{i}\right)_{\ell^{2}} \mapsto H \quad \text { with } T_{\mathcal{W}}^{*}\left(\left\{f_{i}\right\}_{i \in \mathbb{I}}\right)=\sum_{i \in \mathbb{I}} v_{i} f_{i} .
$$


(iii) The operator

$$
S_{\mathcal{W}}: H \mapsto H, \quad S_{\mathcal{W}}(f)=T_{\mathcal{W}}^{*} T_{\mathcal{W}}(f)=\sum_{i \in \mathbb{I}} v_{i}^{2} \pi_{W_{i}}(f)
$$

is called the fusion frame operator of $\left\{\left(W_{i}, v_{i}\right)\right\}_{i \in \mathbb{I}}$.

\section{Relay fusion frames}

In this section, we first introduce the definition of relay fusion frames and then we will show that it also provide an associated analysis and synthesis operator, a frame operator and a dual object.

\subsection{Definition and basic properties of relay fusion frames and their operators}

Definition 6 Let $\left\{K_{i}\right\}_{i \in \mathbb{I}}$ be a sequence of separable Hilbert spaces and $\left\{W_{i}\right\}_{i \in \mathbb{I}}$ be a family of closed subspaces in $H$ and let $\left\{V_{i j}\right\}_{j \in \mathbb{J}_{i}}$ be a family of closed subspaces in $K_{i}$ for each $i \in \mathbb{I}$. Let $\left\{v_{i j}\right\}_{i \in \mathbb{I}, j \in \mathbb{J}_{i}}$ be a family of weights, i.e. $v_{i j}>0$ for each $i \in \mathbb{I}, j \in \mathbb{J}_{i}$, and let $\Lambda_{i} \in \mathcal{B}\left(H, K_{i}\right)$ for each $i \in \mathbb{I}$. Then $\left\{\left(W_{i}, V_{i j}, \Lambda_{i}, v_{i j}\right)\right\}_{i \in \mathbb{I}, j \in \mathbb{J}_{i}}$ is said to be a relay fusion frame, or simply r-fusion frame, if there exist constants $0<\alpha \leq \beta<\infty$ such that

$$
\alpha\|f\|^{2} \leq \sum_{i \in \mathbb{I}} \sum_{j \in \mathbb{J}_{i}} v_{i j}^{2}\left\|\tau_{V_{i j}} \Lambda_{i} \pi_{W_{i}}(f)\right\|^{2} \leq \beta\|f\|^{2}, \quad \forall f \in H .
$$

We call $\alpha$ and $\beta$ the $\mathrm{r}$-fusion frame bounds.

The family $\left\{\left(W_{i}, V_{i j}, \Lambda_{i}, v_{i j}\right)\right\}_{i \in \mathbb{I}, j \in \mathbb{J}_{i}}$ is called an $\alpha$-tight $\mathrm{r}$-fusion frame, if the constants $\alpha$ and $\beta$ can be chosen so that $\alpha=\beta$, a Parseval $\mathrm{r}$-fusion frame provided that $\alpha=\beta=1$. If $\left\{\left(W_{i}, V_{i j}, \Lambda_{i}, v_{i j}\right)\right\}_{i \in \mathbb{I}, j \in \mathbb{J}_{i}}$ satisfies the second inequality in Eq. (2), then it is said to be a Bessel $r$-fusion sequence in $H$ with Bessel r-fusion bound $\beta$.

If we take $K_{i}=H, V_{i j}=W_{i}, \Lambda_{i}=I_{H}$ and $v_{i j}=w_{i}$ for all $i \in \mathbb{I}, j \in \mathbb{J}_{i}$, then we get from Definition 6 the fusion frame $\left\{\left(W_{i}, w_{i}\right)\right\}_{i \in \mathbb{I}}$ for $H$ and thus $r$-fusion frame can be viewed as a generalization of fusion frame.

Similarly, let $W_{i}=H, V_{i j}=K_{i}$ and $v_{i j}=1$ for all $i \in \mathbb{I}, j \in \mathbb{J}_{i}$, then inequality (2) can be restated as the following form which is, as defined in [21], the g-frames:

$$
\alpha\|f\|^{2} \leq \sum_{i \in \mathbb{I}}\left\|\Lambda_{i}(f)\right\|^{2} \leq \beta\|f\|^{2}, \quad \forall f \in H
$$

Consequently, g-frames can be thought of as a special class of $r$-fusion frames. The special case, where $K_{i}=\mathbb{C}, i \in \mathbb{I}$, gives rise to the classical frames.

The representation space employed in classical frame theory and fusion frame theory equal $\ell^{2}(\mathbb{I})$ and $\left(\sum_{i \in \mathbb{I}} \oplus W_{i}\right)_{\ell^{2}}$, respectively. However, in r-fusion frame theory an input signal $f \in H$ is represented by the collection of vector coefficients that can be thought of as to represent the projection onto each subspace of local relay spaces $K_{i}, i \in \mathbb{I}$. Hence, the representation space employed in this framework defined by

$$
\left(\sum_{i \in \mathbb{I}} \sum_{j \in \mathbb{J}_{i}} \oplus V_{i j}\right)_{\ell^{2}}=\left\{\left\{f_{i j}\right\}_{i \in \mathbb{I}, j \in \mathbb{J}_{i}} \mid f_{i j} \in V_{i j} \text { and } \sum_{i \in \mathbb{I}} \sum_{j \in \mathbb{J}_{i}}\left\|f_{i j}\right\|^{2}<\infty\right\},
$$


with inner product given by

$$
\left\langle\left\{f_{i j}\right\}_{i \in \mathbb{I}, j \in \mathbb{J}_{i}},\left\{g_{i j}\right\}_{i \in \mathbb{I}, j \in \mathbb{J}_{i}}\right\rangle=\sum_{i \in \mathbb{I}} \sum_{j \in \mathbb{J}_{i}}\left\langle f_{i j}, g_{i j}\right\rangle,
$$

with respect to the pointwise operations is a Hilbert space.

We can give an intuitive explanation about $r$-fusion frames. Let us assume that we want to transmit the wireless signal $f$ belonging to a vector space $W$ from a transmitter $\mathcal{X}$ to a receiver $\mathcal{Y}$. If the distance between $\mathcal{X}$ and $\mathcal{Y}$ is too far, the wireless signal $f$ will come to nothing before reaching the receiver $\mathcal{Y}$. However, in the case we set up a relay station $\mathcal{Z}$ between $\mathcal{X}$ and $\mathcal{Y}$, this situation will clear away. By transmitting from the relay stations, the restriction that the ordinary receiver and the transmitter cannot be connected due to the distance can be solved.

In the sequel, we will denote $\left\{\left(W_{i}, V_{i j}, \Lambda_{i}, v_{i j}\right)\right\}_{i \in \mathbb{I}, j \in \mathbb{J}}$ by $\mathcal{R}$, simply. We abbreviate r-fusion frame to RFF.

Before define the analysis operator for an RFF, we state the following lemma, which is analogous to Lemma 3.9 in [4].

Lemma 3.1 Let $\mathcal{R}$ be a Bessel $r$-fusion sequence in $H$ with Bessel bound $\beta$. Then, for each sequence $\left\{f_{i j}\right\}_{i \in \mathbb{I}, j \in \mathbb{J}_{i}}$ with $\left\{f_{i j}\right\} \in V_{i j}$ for all $i \in \mathbb{I}, j \in \mathbb{J}_{i}$, the series $\sum_{i \in \mathbb{I}} \sum_{j \in \mathbb{J}_{i}} v_{i j} \pi_{W_{i}} \Lambda_{i}^{*} f_{i j}$ converges unconditionally.

Proof Let $\mathbb{L}$ and $\mathbb{M}$ be fixed finite subsets of $\mathbb{I}$ and $\mathbb{J}_{i}$, respectively. Let

$$
f=\left\{f_{i j}\right\}_{i \in \mathbb{I}, j \in \mathbb{J}_{i}} \in\left(\sum_{i \in \mathbb{I}} \sum_{j \in \mathbb{J}_{i}} \oplus V_{i j}\right)_{\ell^{2}} \text { and } g=\sum_{i \in \mathbb{L}} \sum_{j \in \mathbb{M}} v_{i j} \pi_{W_{i}} \Lambda_{i}^{*} f_{i j} .
$$

Then we have

$$
\begin{aligned}
\|g\| & =\left\|\sum_{i \in \mathbb{L}} \sum_{j \in \mathbb{M}} v_{i j} \pi_{W_{i}} \Lambda_{i}^{*} f_{i j}\right\| \\
& =\sup _{h \in H,\|h\|=1}\left|\left\langle\sum_{i \in \mathbb{L}} \sum_{j \in \mathbb{M}} v_{i j} \pi_{W_{i}} \Lambda_{i}^{*} f_{i j}, h\right\rangle\right| \\
& =\sup _{h \in H,\|h\|=1}\left|\sum_{i \in \mathbb{L}} \sum_{j \in \mathbb{M}}\left\langle f_{i j}, v_{i j} \tau_{V_{i j}} \Lambda_{i} \pi_{W_{i}}(h)\right\rangle\right| \\
& \leq \sup _{h \in H,\|h\|=1}\left(\sum_{i \in \mathbb{L}} \sum_{j \in \mathbb{M}} v_{i j}^{2}\left\|\tau_{V_{i j}} \Lambda_{i} \pi_{W_{i}}(h)\right\|^{2}\right)^{\frac{1}{2}} \cdot\left(\sum_{i \in \mathbb{I}} \sum_{j \in \mathbb{J}_{i}}\left\|f_{i j}\right\|^{2}\right)^{\frac{1}{2}} \\
& \leq \sqrt{\beta}\|f\|,
\end{aligned}
$$

and it follows that $\sum_{i \in \mathbb{I}} \sum_{j \in \mathbb{J}_{i}} v_{i j} \pi_{W_{i}} \Lambda_{i}^{*} f_{i j}$ converges unconditionally (see [8], page 44).

Definition 7 Let $\mathcal{R}$ be an RFF for $\mathrm{H}$. Then the analysis operator for $\mathcal{R}$ is defined by

$$
T_{\mathcal{R}}: H \mapsto\left(\sum_{i \in \mathbb{I}} \sum_{j \in \mathbb{J}_{i}} \oplus V_{i j}\right)_{\ell^{2}} \quad \text { with } T_{\mathcal{R}}(f)=\left\{v_{i j} \tau_{V_{i j}} \Lambda_{i} \pi_{W_{i}}(f)\right\}_{i \in \mathbb{I}, j \in \mathbb{J}}, \forall f \in H
$$

We call the adjoint $T_{\mathcal{R}}^{*}$ of the analysis operator the synthesis operator of $\mathcal{R}$. 
Proposition 3.2 Let $\mathcal{R}$ be an RFF for $H$. Then

$$
T_{\mathcal{R}}^{*}(f)=\sum_{i \in \mathbb{I}} \sum_{j \in \mathbb{J}_{i}} v_{i j} \pi_{W_{i}} \Lambda_{i}^{*} f_{i j}, \quad \forall f=\left\{f_{i j}\right\}_{i \in \mathbb{I}, j \in \mathbb{J}_{i}} \in\left(\sum_{i \in \mathbb{I}} \sum_{j \in \mathbb{J}_{i}} \oplus V_{i j}\right)_{\ell^{2}} .
$$

Proof Let $g \in H$ and $f=\left\{f_{i j}\right\}_{i \in \mathbb{I}, j \in \mathbb{J}_{i}} \in\left(\sum_{i \in \mathbb{I}} \sum_{j \in \mathbb{J}_{i}} \oplus V_{i j}\right)_{\ell^{2}}$. Then we compute

$$
\begin{aligned}
\left\langle T_{\mathcal{R}}(g), f\right\rangle & =\left\langle\left\{v_{i j} \tau_{V_{i j}} \Lambda_{i} \pi_{W_{i}}(g)\right\}_{i \in \mathbb{I}, j \in \mathbb{J}_{i}},\left\{f_{i j}\right\}_{i \in \mathbb{I}, j \in \mathbb{J}_{i}}\right\rangle \\
& =\sum_{i \in \mathbb{I}} \sum_{j \in \mathbb{J}_{i}}\left\langle g, v_{i j} \pi_{W_{i}} \Lambda_{i}^{*}\left(f_{i j}\right)\right\rangle=\left\langle g, T_{\mathcal{R}}^{*}(f)\right\rangle .
\end{aligned}
$$

In an analogous way as in frame and fusion frame theory we can give the following wellknown relations between an RFF and the associated analysis and synthesis operator.

Theorem 3.3 The following assertions are equivalent:

(i) $\mathcal{R}$ is an RFF for $H$.

(ii) The analysis operator $T_{\mathcal{R}}$ is injective and has closed range.

(iii) The synthesis operator $T_{\mathcal{R}}^{*}$ is bounded, linear and surjective.

Proof (i) $\Rightarrow$ (ii) For all $f \in H$, we have

$$
\left\|T_{\mathcal{R}}(f)\right\|^{2}=\sum_{i \in \mathbb{I}} \sum_{j \in \mathbb{J}_{i}} v_{i j}^{2}\left\|\tau_{V_{i j}} \Lambda_{i} \pi_{W_{i}}(f)\right\|^{2} \geq \alpha\|f\|^{2},
$$

which implies that $T_{\mathcal{R}}$ is injective and has closed range.

(ii) $\Rightarrow$ (i) This is obvious.

(ii) $\Leftrightarrow$ (iii) This follows immediately from the operator-theoretics results of Hilbert spaces.

By composing $T_{\mathcal{R}}$ and $T_{\mathcal{R}}^{*}$, we obtain the frame operator for $\mathcal{R}$.

Definition 8 Let $\mathcal{R}$ be an RFF for $\mathrm{H}$. Then the frame operator $S_{\mathcal{R}}$ for $\mathcal{R}$ is defined by

$$
S_{\mathcal{R}}(f)=T_{\mathcal{R}}^{*} T_{\mathcal{R}}(f)=\sum_{i \in \mathbb{I}} \sum_{j \in \mathbb{J}_{i}} v_{i j}^{2} \pi_{W_{i}} \Lambda_{i}^{*} \tau_{V_{i j}} \Lambda_{i} \pi_{W_{i}}(f), \quad \forall f \in H .
$$

To prove Proposition 3.5 we need the following theorem that gives the relation between a Bessel $r$-fusion sequence and the synthesis operator $T_{\mathcal{R}}^{*}$.

Theorem 3.4 $\mathcal{R}$ is a Bessel $r$-fusion sequence in $H$ with bound $\beta$ if and only if the map

$$
\left(\left\{f_{i j}\right\}_{i \in \mathbb{I}, j \in \mathbb{I}_{i}}\right) \mapsto \sum_{i \in \mathbb{I}} \sum_{j \in \mathbb{J}_{i}} v_{i j} \pi_{W_{i}} \Lambda_{i}^{*} f_{i j}
$$

is a well-defined bounded operator from $\left(\sum_{i \in \mathbb{I}} \sum_{j \in \mathbb{I}_{i}} \oplus V_{i j}\right)_{\ell^{2}}$ to $H$ and its norm is less than or equal to $\sqrt{\beta}$. 
Proof First assume that $\mathcal{R}$ is a Bessel $\mathrm{r}$-fusion sequence for $\mathrm{H}$ with bound $\beta$. By Lemma 3.1, the series $\sum_{i \in \mathbb{I}} \sum_{j \in \mathbb{J}_{i}} v_{i j} \pi_{W_{i}} \Lambda_{i}^{*} f_{i j}$ is convergent. Thus $T_{\mathcal{R}}^{*}\left(\left\{f_{i j}\right\}_{i \in \mathbb{I}, j \in \mathbb{J}_{i}}\right)$ is well defined. A simple calculation as in Lemma 3.1 shows that $T_{\mathcal{R}}^{*}$ is bounded and that $\left\|T_{\mathcal{R}}^{*}\right\| \leq \sqrt{\beta}$.

For the opposite implication, suppose that $T_{\mathcal{R}}^{*}$ is well defined and that $\left\|T_{\mathcal{R}}^{*}\right\| \leq \sqrt{\beta}$. Then

$$
\begin{aligned}
& \sum_{i \in \mathbb{I}} \sum_{j \in \mathbb{J}_{i}} v_{i j}^{2}\left\|\tau_{V_{i j}} \Lambda_{i} \pi_{W_{i}}(f)\right\|^{2} \\
& =\sum_{i \in \mathbb{I}} \sum_{j \in \mathbb{J}_{i}} v_{i j}^{2}\left(\pi_{W_{i}} \Lambda_{i}^{*} \tau_{V_{i j}} \Lambda_{i} \pi_{W_{i}}(f), f\right\rangle \\
& =\left\langle T_{\mathcal{R}}^{*}\left(\left\{v_{i j} \tau_{V_{i j}} \Lambda_{i} \pi_{W_{i}}(f)\right\}_{i \in \mathbb{I}, j \in \mathbb{J}_{i}}\right), f\right\rangle \\
& \quad \leq\left(\sum_{i \in \mathbb{I}} \sum_{j \in \mathbb{J}_{i}} v_{i j}^{2}\left\|\tau_{V_{i j}} \Lambda_{i} \pi_{W_{i}}(f)\right\|^{2}\right)^{\frac{1}{2}}\left\|T_{\mathcal{R}}^{*}\right\|\|f\| .
\end{aligned}
$$

Now solving for $\left(\sum_{i \in \mathbb{I}} \sum_{j \in \mathbb{J}_{i}} v_{i j}^{2}\left\|\tau_{V_{i j}} \Lambda_{i} \pi_{W_{i}}(f)\right\|^{2}\right)^{\frac{1}{2}}$ yields

$$
\left(\sum_{i \in \mathbb{I}} \sum_{j \in \mathbb{J}_{i}} v_{i j}^{2}\left\|\tau_{V_{i j}} \Lambda_{i} \pi_{W_{i}}(f)\right\|^{2}\right)^{\frac{1}{2}} \leq\left\|T_{\mathcal{R}}^{*}\right\|\|f\| \leq \sqrt{\beta}\|f\| .
$$

Given an RFF, Proposition 3.5 states some of the important properties of frame operator $S_{\mathcal{R}}$.

Proposition 3.5 Let $\mathcal{R}$ be an RFF with frame bounds $\alpha$ and $\beta$. Then the frame operator for $\mathcal{R}$ is a bounded, positive, self-adjoint, invertible operator on $H$ with $\alpha I_{H} \leq S_{\mathcal{R}} \leq \beta I_{H}$.

Proof $S_{\mathcal{R}}$ is bounded as a composition of two bounded operators. By Theorem 3.4,

$$
\left\|S_{\mathcal{R}}\right\|=\left\|T_{\mathcal{R}}^{*} T_{\mathcal{R}}\right\|=\left\|T_{\mathcal{R}}^{*}\right\|^{2} \leq \beta .
$$

Since $S_{\mathcal{R}}^{*}=\left(T_{\mathcal{R}}^{*} T_{\mathcal{R}}\right)^{*}=T_{\mathcal{R}}^{*} T_{\mathcal{R}}=S_{\mathcal{R}}$, the operator $S_{\mathcal{R}}$ is self-adjoint. The inequality (2) means that

$$
\alpha\|f\|^{2} \leq\left\langle S_{\mathcal{R}}(f), f\right\rangle \leq \beta\|f\|^{2}, \quad \forall f \in H .
$$

This shows that $\alpha I_{H} \leq S_{\mathcal{R}} \leq \beta I_{H}$ and hence $S_{\mathcal{R}}$ is a positive, invertible operator on $H$.

Proposition 3.6 Let $\mathcal{R}$ be an RFF for $H$ with frame operator $S_{\mathcal{R}}$, we have then, for all $f \in H$,

$$
f=\sum_{i \in \mathbb{I}} \sum_{j \in \mathbb{J}_{i}} v_{i j}^{2} S_{\mathcal{R}}^{-1} \pi_{W_{i}} \Lambda_{i}^{*} \tau_{V_{i j}} \Lambda_{i} \pi_{W_{i}}(f)=\sum_{i \in \mathbb{I}} \sum_{j \in \mathbb{J}_{i}} v_{i j}^{2} \pi_{W_{i}} \Lambda_{i}^{*} \tau_{V_{i j}} \Lambda_{i} \pi_{W_{i}} S_{\mathcal{R}}^{-1}(f) .
$$

Proof Since $S_{\mathcal{R}}$ is invertible, for all $f \in H$ we have

$$
\begin{aligned}
f & =S_{\mathcal{R}}^{-1} S_{\mathcal{R}}(f)=\sum_{i \in \mathbb{I}} \sum_{j \in \mathbb{J}_{i}} v_{i j}^{2} S_{\mathcal{R}}^{-1} \pi_{W_{i}} \Lambda_{i}^{*} \tau_{V_{i j}} \Lambda_{i} \pi_{W_{i}}(f) \\
& =S_{\mathcal{R}} S_{\mathcal{R}}^{-1}(f)=\sum_{i \in \mathbb{I}} \sum_{j \in \mathbb{J}_{i}} v_{i j}^{2} \pi_{W_{i}} \Lambda_{i}^{*} \tau_{V_{i j}} \Lambda_{i} \pi_{W_{i}} S_{\mathcal{R}}^{-1}(f) .
\end{aligned}
$$


The following theorem gives a sufficient condition such that two Bessel $r$-fusion sequence become RFFs in terms of their analysis operators.

Theorem 3.7 Let $\mathcal{R}_{1}=\left\{\left(W_{i}, V_{i j}, \Lambda_{i}, v_{i j}\right)\right\}_{i \in \mathbb{I}, j \in \mathbb{J}_{i}}$ and $\mathcal{R}_{2}=\left\{\left(W_{i}^{\prime}, V_{i j}^{\prime}, \Lambda_{i}^{\prime}, v_{i j}^{\prime}\right)\right\}_{i \in \mathbb{I}, j \in \mathbb{J}_{i}}$ be two Bessel r-fusion sequence for $H$ with bounds $\beta_{1}$ and $\beta_{2}$, respectively. Let $T_{\mathcal{R}_{1}}$ and $T_{\mathcal{R}_{2}}$ be their analysis operators such that $T_{\mathcal{R}_{2}}^{*} T_{\mathcal{R}_{1}}=I_{H}$. Then both $\mathcal{R}_{1}$ and $\mathcal{R}_{2}$ are RFFs.

Proof For all $f \in H$, we have

$$
\begin{aligned}
\|f\|^{4} & =\left(\left\langle T_{\mathcal{R}_{1}}(f), T_{\mathcal{R}_{2}}(f)\right\rangle\right)^{2} \\
& \leq\left\|T_{\mathcal{R}_{1}}(f)\right\|^{2}\left\|T_{\mathcal{R}_{2}}(f)\right\|^{2} \\
& =\left(\sum_{i \in \mathbb{I}} \sum_{j \in \mathbb{J}_{i}} v_{i j}^{2}\left\|\tau_{V_{i j}} \Lambda_{i} \pi_{W_{i}}(f)\right\|^{2}\right)\left(\sum_{i \in \mathbb{I}} \sum_{j \in \mathbb{J}_{i}} v_{i j}^{\prime 2}\left\|\tau_{V_{i j}^{\prime}} \Lambda_{i}^{\prime} \pi_{W_{i}^{\prime}}(f)\right\|^{2}\right) \\
& \leq\left(\sum_{i \in \mathbb{I}} \sum_{j \in \mathbb{J}_{i}} v_{i j}^{2}\left\|\tau_{V_{i j}} \Lambda_{i} \pi_{W_{i}}(f)\right\|^{2}\right) \beta_{2}\|f\|^{2} .
\end{aligned}
$$

This yields

$$
\frac{1}{\beta_{2}}\|f\|^{2} \leq \sum_{i \in \mathbb{I}} \sum_{j \in \mathbb{J}_{i}} v_{i j}^{2}\left\|\tau_{V_{i j}} \Lambda_{i} \pi_{W_{i}}(f)\right\|^{2} .
$$

Similarly we obtain a lower bound for $\mathcal{R}_{2}$.

\subsection{Duality of relay fusion frames}

To define the dual frames for RFFs, we need the following technical lemma.

Lemma 3.8 (see [10]) Let $A \in \mathcal{B}(H)$ and $V \subseteq H$ be a closed subspace. Then

$$
\pi_{V} A^{*}=\pi_{V} A^{*} \pi \overline{A V}
$$

\subsubsection{Global relay dual of relay fusion frames}

Let $\mathcal{R}=\left\{\left(W_{i}, V_{i j}, \Lambda_{i}, v_{i j}\right)\right\}_{i \in \mathbb{I}, j \in \mathbb{J}_{i}}$ be an RFF for $\mathrm{H}$. We consider global relay space $\mathcal{K}=$ $\left(\sum_{i \in \mathbb{I}} \oplus K_{i}\right)_{\ell^{2}}$ and let $\mathcal{F}_{\mathcal{K}}$ be a frame for $\mathcal{K}$, where every $K_{i}$ is local relay space. We use $S_{\mathcal{F}_{\mathcal{K}}}$ to denote the frame operator for $\mathcal{K}$. Let $\underline{V}_{i j}=S_{\mathcal{F}_{\mathcal{K}}}^{-1} V_{i j}$ and $\underline{\Lambda}_{i}=S_{\mathcal{F}_{\mathcal{K}}}^{-1} \tau_{V_{i j}} \Lambda_{i}$. We now prove that $\underline{\mathcal{R}}=\left\{\left(W_{i}, \underline{V}_{i j}, \underline{\Lambda}_{i}, v_{i j}\right)\right\}_{i \in \mathbb{I}, j \in \mathbb{J}_{i}}$ is an RFF for $H$ and we call $\underline{\mathcal{R}}$ the global relay dual RFF of $\mathcal{R}$.

Theorem 3.9 Let $\mathcal{R}$ be an RFF for $H$. Then $\underline{\mathcal{R}}$ is an RFF for $H$ and, for all $f \in H$,

$$
f=\sum_{i \in \mathbb{I}} \sum_{j \in \mathbb{J}_{i}} v_{i j}^{2} S_{\mathcal{R}}^{-1} \pi_{W_{i}} \underline{\Lambda}_{i}^{*} \underline{\Lambda}_{i} \pi_{W_{i}}(f)=\sum_{i \in \mathbb{I}} \sum_{j \in \mathbb{J}_{i}} v_{i j}^{2} \pi_{W_{i}} \underline{\Lambda}_{i}^{*} \underline{\Lambda}_{i} \pi_{W_{i}} S_{\underline{\mathcal{R}}}^{-1}(f) .
$$

Proof We first prove the upper bound. For each $f \in H$, we have

$$
\sum_{i \in \mathbb{I}} \sum_{j \in \mathbb{J}_{i}} v_{i j}^{2}\left\|\tau_{S_{\mathcal{F}_{\mathcal{K}}}^{-1}} V_{i j} S_{\mathcal{F}_{\mathcal{K}}}^{-1} \tau_{V_{i j}} \Lambda_{i} \pi_{W_{i}}(f)\right\|^{2}=\sum_{i \in \mathbb{I}} \sum_{j \in \mathbb{J}_{i}} v_{i j}^{2}\left\|S_{\mathcal{F}_{\mathcal{K}}}^{-1} \tau_{V_{i j}} \Lambda_{i} \pi_{W_{i}}(f)\right\|^{2}
$$




$$
\leq\left\|S_{\mathcal{F}_{\mathcal{K}}}^{-1}\right\|^{2} \beta\|f\|^{2}
$$

Now we obtain a lower bound for $\underline{\mathcal{R}}$. We compute

$$
\begin{aligned}
\sum_{i \in \mathbb{I}} \sum_{j \in \mathbb{J}_{i}} v_{i j}^{2}\left\|\tau_{S_{\mathcal{F}_{\mathcal{K}}} V_{i j}} S_{\mathcal{F}_{\mathcal{K}}}^{-1} \tau_{V_{i j}} \Lambda_{i} \pi_{W_{i}}(f)\right\|^{2} & =\sum_{i \in \mathbb{I}} \sum_{j \in \mathbb{J}_{i}} v_{i j}^{2}\left\|S_{\mathcal{F}_{\mathcal{K}}}^{-1} \tau_{V_{i j}} \Lambda_{i} \pi_{W_{i}}(f)\right\|^{2} \\
& \geq \sum_{i \in \mathbb{I}} \sum_{j \in \mathbb{J}_{i}} v_{i j}^{2} \frac{1}{\left\|S_{\mathcal{F}_{\mathcal{K}}}\right\|^{2}}\left\|\tau_{V_{i j}} \Lambda_{i} \pi_{W_{i}}(f)\right\|^{2} \\
& \geq \frac{\alpha}{\left\|S_{\mathcal{F}_{\mathcal{K}}}\right\|^{2}}\|f\|^{2} .
\end{aligned}
$$

Further, since $S_{\widetilde{\mathcal{R}}}$ is invertible, for all $f \in H$ we have

$$
\begin{aligned}
f & =S_{\underline{\mathcal{R}}}^{-1} S_{\underline{\mathcal{R}}}(f)=S_{\underline{\mathcal{R}}} S_{\underline{\mathcal{R}}}^{-1}(f) \\
& =\sum_{i \in \mathbb{I}} \sum_{j \in \mathbb{J}_{i}} v_{i j}^{2} S_{\mathcal{\mathcal { R }}}^{-1} \pi_{W_{i}} \underline{\Lambda}_{i}^{*} \tau_{\underline{V}_{i j}} \underline{\Lambda}_{i} \pi_{W_{i}}(f) \\
& =\sum_{i \in \mathbb{I}} \sum_{j \in \mathbb{J}_{i}} v_{i j}^{2} S_{\underline{\mathcal{R}}}^{-1} \pi_{W_{i}} \Lambda_{i}^{*} \tau_{V_{i j}} S_{i}^{-1} \tau_{S_{i}^{-1} V_{i j}} S_{i}^{-1} \tau_{V_{i j}} \Lambda_{i} \pi_{W_{i}}(f) \\
& =\sum_{i \in \mathbb{I}} \sum_{j \in \mathbb{J}_{i}} v_{i j}^{2} S_{\underline{\mathcal{R}}}^{-1} \pi_{W_{i}} \underline{\Lambda}_{i}^{*} \underline{\Lambda}_{i} \pi_{W_{i}}(f) \\
& =\sum_{i \in \mathbb{I}} \sum_{j \in \mathbb{J}_{i}} v_{i j}^{2} \pi_{W_{i}} \underline{\Lambda}_{i}^{*} \underline{\Lambda}_{i} \pi_{W_{i}} S_{\underline{\mathcal{R}}}^{-1}(f) .
\end{aligned}
$$

\subsubsection{Local relay dual of relay fusion frames}

Let $\widetilde{V}_{i j}=S_{i}^{-1} V_{i j}$ and $\widetilde{\Lambda}_{i}=S_{i}^{-1} \tau_{V_{i j}} \Lambda_{i}$, where $S_{i}$ denote the frame operators with respect to $K_{i}$ for each $i \in \mathbb{I}$ and we call every $S_{i}$ local relay frame operator. We now prove that $\widetilde{\mathcal{R}}=$ $\left\{\left(W_{i}, \widetilde{V}_{i j}, \widetilde{\Lambda}_{i}, v_{i j}\right)\right\}_{i \in \mathbb{I}, j \in \mathbb{J}_{i}}$ is also an RFF for $H$ and we call $\widetilde{\mathcal{R}}$ the local relay dual RFF of $\mathcal{R}$.

Theorem 3.10 Let $\mathcal{R}$ be an RFF for $H$. Then $\widetilde{\mathcal{R}}$ is an RFF for $H$ and, for all $f \in H$,

$$
f=\sum_{i \in \mathbb{I}} \sum_{j \in \mathbb{J}_{i}} v_{i j}^{2} S_{\widetilde{\mathcal{R}}}^{-1} \pi_{W_{i}} \tilde{\Lambda}_{i}^{*} \tilde{\Lambda}_{i} \pi_{W_{i}}(f)=\sum_{i \in \mathbb{I}} \sum_{j \in \mathbb{J}_{i}} v_{i j}^{2} \pi_{W_{i}} \tilde{\Lambda}_{i}^{*} \tilde{\Lambda}_{i} \pi_{W_{i}} S_{\widetilde{\mathcal{R}}}^{-1}(f)
$$

Proof It is easy to show that, for all $f \in H$,

$$
\begin{aligned}
\sum_{i \in \mathbb{I}} \sum_{j \in \mathbb{J}_{i}} v_{i j}^{2}\left\|\tau_{S_{i}^{-1} V_{i j}} S_{i}^{-1} \tau_{V_{i j}} \Lambda_{i} \pi_{W_{i}}(f)\right\|^{2} & =\sum_{i \in \mathbb{I}} \sum_{j \in \mathbb{J}_{i}} v_{i j}^{2}\left\|S_{i}^{-1} \tau_{V_{i j}} \Lambda_{i} \pi_{W_{i}}(f)\right\|^{2} \\
& \leq \max _{i \in \mathbb{I}}\left\{\left\|S_{i}^{-1}\right\|^{2} \beta\right\}\|f\|^{2} .
\end{aligned}
$$

Now we obtain a lower bound for $\widetilde{\mathcal{R}}$. We compute

$$
\begin{aligned}
\sum_{i \in \mathbb{I}} \sum_{j \in \mathbb{J}_{i}} v_{i j}^{2}\left\|\tau_{S_{i}^{-1} V_{i j}} S_{i}^{-1} \tau_{V_{i j}} \Lambda_{i} \pi_{W_{i}}(f)\right\|^{2} & =\sum_{i \in \mathbb{I}} \sum_{j \in \mathbb{J}_{i}} v_{i j}^{2}\left\|S_{i}^{-1} \tau_{V_{i j}} \Lambda_{i} \pi_{W_{i}}(f)\right\|^{2} \\
& \geq \sum_{i \in \mathbb{I}} \sum_{j \in \mathbb{J}_{i}} v_{i j}^{2} \frac{1}{\left\|S_{i}\right\|^{2}}\left\|\tau_{V_{i j}} \Lambda_{i} \pi_{W_{i}}(f)\right\|^{2}
\end{aligned}
$$




$$
\geq \min _{i \in \mathbb{I}}\left\{\frac{\alpha}{\left\|S_{i}\right\|^{2}}\right\}\|f\|^{2}
$$

Further, since $S_{\widetilde{\mathcal{R}}}$ is invertible, for all $f \in H$ we have

$$
\begin{aligned}
& f=S_{\widetilde{\mathcal{R}}}^{-1} S_{\widetilde{\mathcal{R}}}(f)=S_{\widetilde{\mathcal{R}}} S_{\widetilde{\mathcal{R}}}^{-1}(f) \\
& =\sum_{i \in \mathbb{I}} \sum_{j \in \mathbb{J}_{i}} v_{i j}^{2} S_{\widetilde{\mathcal{R}}}^{-1} \pi_{W_{i}} \widetilde{\Lambda}_{i}^{*} \tau_{\widetilde{V}_{i j}} \widetilde{\Lambda}_{i} \pi_{W_{i}}(f) \\
& =\sum_{i \in \mathbb{I}} \sum_{j \in \mathbb{J}_{i}} v_{i j}^{2} S_{\widetilde{\mathcal{R}}}^{-1} \pi_{W_{i}} \Lambda_{i}^{*} \tau_{V_{i j}} S_{i}^{-1} \tau_{S_{i}^{-1} V_{i j}} S_{i}^{-1} \tau_{V_{i j}} \Lambda_{i} \pi_{W_{i}}(f) \\
& =\sum_{i \in \mathbb{I}} \sum_{j \in \mathbb{J}_{i}} v_{i j}^{2} S_{\widetilde{\mathcal{R}}}^{-1} \pi_{W_{i}} \widetilde{\Lambda}_{i}^{*} \widetilde{\Lambda}_{i} \pi_{W_{i}}(f) \\
& =\sum_{i \in \mathbb{I}} \sum_{j \in \mathbb{J}_{i}} v_{i j}^{2} \pi_{W_{i}} \tilde{\Lambda}_{i}^{*} \tilde{\Lambda}_{i} \pi_{W_{i}} S_{\widetilde{\mathcal{R}}}^{-1}(f) .
\end{aligned}
$$

Remark 3.11 Recall that there are always many different frames for global relay space $\mathcal{K}$ and local relay space $K_{i}$, respectively. For this reason, the global relay dual RFF $\underline{\mathcal{R}}$ and local relay dual RFF $\widetilde{\mathcal{R}}$ are not unique.

\subsubsection{Canonical dual of relay fusion frames}

Now let $\widehat{W}_{i}=S_{\mathcal{R}}^{-1} W_{i}$ and $\widehat{\Lambda}_{i}=\Lambda_{i} \pi_{W_{i}} S_{\mathcal{R}}^{-1}$, where $S_{\mathcal{R}}$ is the frame operator for $\mathcal{R}$. We prove that $\widehat{\mathcal{R}}=\left\{\left(\widehat{W}_{i}, V_{i j}, \widehat{\Lambda}_{i}, v_{i j}\right)\right\}_{i \in \mathbb{I}, j \in \mathbb{J}_{i}}$ is also an RFF for $H$ and we call $\widehat{\mathcal{R}}$ the canonical dual RFF of $\mathcal{R}$ for $H$.

Theorem 3.12 Let $\mathcal{R}$ be an RFF for $H$. Then $\widehat{\mathcal{R}}$ is an RFF for $H$.

Proof For all $f \in H$, we have

$$
\begin{aligned}
\sum_{i \in \mathbb{I}} \sum_{j \in \mathbb{J}_{i}} v_{i j}^{2}\left\|\tau_{V_{i j}} \Lambda_{i} \pi_{W_{i}} S_{\mathcal{R}}^{-1} \pi_{S_{\mathcal{R}}^{-1} W_{i}}(f)\right\|^{2} & =\sum_{i \in \mathbb{I}} \sum_{j \in \mathbb{J}_{i}} v_{i j}^{2}\left\|\tau_{V_{i j}} \Lambda_{i} \pi_{W_{i}} S_{\mathcal{R}}^{-1}(f)\right\|^{2} \\
& \leq\left\|S_{\mathcal{R}}^{-1}\right\|^{2} \beta\|f\|^{2} .
\end{aligned}
$$

Now we obtain a lower bound for $\widehat{\mathcal{R}}$. We compute

$$
\begin{aligned}
\|f\|^{4} & =\left|\left\langle\sum_{i \in \mathbb{I}} \sum_{j \in \mathbb{J}_{i}} v_{i j}^{2} \pi_{W_{i}} \Lambda_{i}^{*} \tau_{V_{i j}} \Lambda_{i} \pi_{W_{i}} S_{\mathcal{R}}^{-1}(f), f\right)\right|^{2} \\
& =\left|\sum_{i \in \mathbb{I}} \sum_{j \in \mathbb{J}_{i}} v_{i j}^{2}\left(\tau_{V_{i j}} \Lambda_{i} \pi_{W_{i}} S_{\mathcal{R}}^{-1}(f), \tau_{V_{i j}} \Lambda_{i} \pi_{W_{i}} f\right\rangle\right|^{2} \\
& \leq\left(\sum_{i \in \mathbb{I}} \sum_{j \in \mathbb{J}_{i}} v_{i j}^{2}\left\|\tau_{V_{i j}} \Lambda_{i} \pi_{W_{i}} S_{\mathcal{R}}^{-1} \pi_{S_{\mathcal{R}}^{-1} W_{i}}(f)\right\|^{2}\right)\left(\sum_{i \in \mathbb{I}} \sum_{j \in \mathbb{J}_{i}} v_{i j}^{2}\left\|\tau_{V_{i j}} \Lambda_{i} \pi_{W_{i}}(f)\right\|^{2}\right) \\
& \leq\left(\sum_{i \in \mathbb{I}} \sum_{j \in \mathbb{J}_{i}} v_{i j}^{2}\left\|\tau_{V_{i j}} \Lambda_{i} \pi_{W_{i}} S_{\mathcal{R}}^{-1} \pi_{S_{\mathcal{R}}^{-1} W_{i}}(f)\right\|^{2}\right) \beta\|f\|^{2},
\end{aligned}
$$


which implies that

$$
\frac{1}{\beta}\|f\|^{2} \leq \sum_{i \in \mathbb{I}} \sum_{j \in \mathbb{J}_{i}} v_{i j}^{2}\left\|\tau_{V_{i j}} \Lambda_{i} \pi_{W_{i}} S_{\mathcal{R}}^{-1} \pi_{S_{\mathcal{R}}^{-1} W_{i}}(f)\right\|^{2}
$$

Theorem 3.13 Let $\mathcal{R}$ be an RFF for $H$ with frame operator $S_{\mathcal{R}}$ and let $\widehat{\mathcal{R}}$ be the canonical dual RFF of $\mathcal{R}$ with frame operator $S_{\widehat{\mathcal{R}}}$. Then $S_{\mathcal{R}} S_{\widehat{\mathcal{R}}}=I_{H}$ and $T_{\mathcal{R}}^{*} T_{\widehat{\mathcal{R}}}=I_{H}$ and, for all $f \in H$,

$$
f=\sum_{i \in \mathbb{I}} \sum_{j \in \mathbb{J}_{i}} v_{i j}^{2} \pi_{W_{i}} \Lambda_{i}^{*} \tau_{V_{i j}} \widehat{\Lambda}_{i} \pi_{\widehat{W}_{i}}(f)=\sum_{i \in \mathbb{I}} \sum_{j \in \mathbb{J}_{i}} v_{i j}^{2} \pi_{\widehat{W}_{i}} \widehat{\Lambda}_{i}^{*} \tau_{V_{i j}} \Lambda_{i} \pi_{W_{i}}(f) .
$$

Proof For all $f \in H$, we obtain

$$
\begin{aligned}
S_{\mathcal{R}} S_{\widehat{\mathcal{R}}}(f) & =S_{\mathcal{R}} \sum_{i \in \mathbb{I}} \sum_{j \in \mathbb{J}_{i}} v_{i j}^{2} \pi_{S_{\mathcal{R}}^{-1} W_{i}} S_{\mathcal{R}}^{-1} \pi_{W_{i}} \Lambda_{i}^{*} \tau_{V_{i j}} \Lambda_{i} \pi_{W_{i}} S_{\mathcal{R}}^{-1} \pi_{S_{\mathcal{R}}^{-1} W_{i}}(f) \\
& =S_{\mathcal{R}} \sum_{i \in \mathbb{I}} \sum_{j \in \mathbb{J}_{i}} v_{i j}^{2} S_{\mathcal{R}}^{-1} \pi_{W_{i}} \Lambda_{i}^{*} \tau_{V_{i j}} \Lambda_{i} \pi_{W_{i}} S_{\mathcal{R}}^{-1}(f) \\
& =\sum_{i \in \mathbb{I}} \sum_{j \in \mathbb{J}_{i}} v_{i j}^{2} \pi_{W_{i}} \Lambda_{i}^{*} \tau_{V_{i j}} \Lambda_{i} \pi_{W_{i}} S_{\mathcal{R}}^{-1}(f) \\
& =S_{\mathcal{R}} S_{\mathcal{R}}^{-1}(f) \\
& =f
\end{aligned}
$$

and

$$
\begin{aligned}
T_{\mathcal{R}}^{*} T_{\widehat{\mathcal{R}}}(f) & =T_{\mathcal{R}}^{*}\left(\left\{v_{i j} \tau_{V_{i j}} \Lambda_{i} \pi_{W_{i}} S_{\mathcal{R}}^{-1} \pi_{S_{\mathcal{R}}^{-1} W_{i}}(f)\right\}_{i \in \mathbb{I}, j \in \mathbb{J}_{i}}\right) \\
& =T_{\mathcal{R}}^{*}\left(\left\{v_{i j} \tau_{V_{i j}} \Lambda_{i} \pi_{W_{i}} S_{\mathcal{R}}^{-1}(f)\right\}_{i \in \mathbb{I}, j \in \mathbb{J}_{i}}\right) \\
& =T_{\mathcal{R}}^{*} T_{\mathcal{R}} S_{\mathcal{R}}^{-1}(f) \\
& =f .
\end{aligned}
$$

The last assertion of the theorem follows from the previous steps of the proof.

Moreover, the canonical dual RFFs give rise to expansion coefficients with the minimal norm.

Theorem 3.14 Let $\mathcal{R}$ be an RFF with canonical dual RFF $\widehat{\mathcal{R}}$. Then, for any $g_{i j} \in V_{i j}$ satisfying $f=\sum_{i \in \mathbb{I}} \sum_{j \in \mathbb{J}_{i}} v_{i j}^{2} \pi_{W_{i}} \Lambda_{i}^{*} g_{i j}$, we have

$$
\sum_{i \in \mathbb{I}} \sum_{j \in \mathbb{J}_{i}}\left\|g_{i j}\right\|^{2}=\sum_{i \in \mathbb{I}} \sum_{j \in \mathbb{J}_{i}} v_{i j}^{2}\left\|\tau_{V_{i j}} \widehat{\Lambda}_{i} \pi_{\widehat{W}_{i}}(f)\right\|^{2}+\sum_{i \in \mathbb{I}} \sum_{j \in \mathbb{J}_{i}}\left\|g_{i j}-v_{i j}^{2} \tau_{V_{i j}} \widehat{\Lambda}_{i} \pi_{\widehat{W}_{i}}(f)\right\|^{2} .
$$

Proof We compute

$$
\begin{aligned}
\sum_{i \in \mathbb{I}} \sum_{j \in \mathbb{J}_{i}} v_{i j}^{2}\left\|\tau_{V_{i j}} \widehat{\Lambda}_{i} \pi_{\widehat{W_{i}}}(f)\right\|^{2} & =\left\langle S_{\widehat{\mathcal{R}}}(f), f\right\rangle \\
& =\left\langle f, S_{\mathcal{R}}^{-1}(f)\right\rangle
\end{aligned}
$$




$$
\begin{aligned}
& =\sum_{i \in \mathbb{I}} \sum_{j \in \mathbb{J}_{i}} v_{i j}^{2}\left\langle\pi_{W_{i}} \Lambda_{i}^{*} g_{i j}, S_{\mathcal{R}}^{-1}(f)\right\rangle \\
& =\sum_{i \in \mathbb{I}} \sum_{j \in \mathbb{J}_{i}} v_{i j}^{2}\left\langle g_{i j}, \tau_{V_{i j}} \Lambda_{i} \pi_{W_{i}} S_{\mathcal{R}}^{-1}(f)\right\rangle \\
& =\sum_{i \in \mathbb{I}} \sum_{j \in \mathbb{J}_{i}} v_{i j}^{2}\left|g_{i j}, \tau_{V_{i j}} \widehat{\Lambda}_{i} \pi_{\widehat{W}_{i}}(f)\right\rangle \\
& =\sum_{i \in \mathbb{I}} \sum_{j \in \mathbb{J}_{i}} v_{i j}^{2}\left\langle\tau_{V_{i j}} \widehat{\Lambda}_{i} \pi_{\widehat{W}}(f), g_{i j}\right\rangle,
\end{aligned}
$$

which finishes the proof.

Example 3.15 In classical frame theory we can always construction a Parseval frame by applying $S_{\mathcal{F}}^{-\frac{1}{2}}$, where $S_{\mathcal{F}}$ denote the frame operator of frame $\mathcal{F}$. For the situation of RFFs is similarly. In fact, for all $f \in H$,

$$
\begin{aligned}
\|f\|^{2}=\left\langle S_{\mathcal{R}}^{-\frac{1}{2}} S_{\mathcal{R}} S_{\mathcal{R}}^{-\frac{1}{2}}(f), f\right\rangle & =\left\langle\sum_{i \in \mathbb{I}} \sum_{j \in \mathbb{J}_{i}} v_{i j}^{2} S_{\mathcal{R}}^{-\frac{1}{2}} \pi_{W_{i}} \Lambda_{i}^{*} \tau_{V_{i j}} \Lambda_{i} \pi_{W_{i}} S_{\mathcal{R}}^{-\frac{1}{2}}(f), f\right\rangle \\
& =\sum_{i \in \mathbb{I}} \sum_{j \in \mathbb{J}_{i}} v_{i j}^{2}\left\|\tau_{V_{i j}} \Lambda_{i} \pi_{W_{i}} S_{\mathcal{R}}^{-\frac{1}{2}}(f)\right\|^{2} \\
& =\sum_{i \in \mathbb{I}} \sum_{j \in \mathbb{J}_{i}} v_{i j}^{2}\left\|\tau_{V_{i j}} \Lambda_{i} \pi_{W_{i}} S_{\mathcal{R}}^{-\frac{1}{2}} \pi_{S_{\mathcal{R}}^{-\frac{1}{2}} W_{i}}(f)\right\|^{2} ;
\end{aligned}
$$

therefore, $\left\{\left(S_{\mathcal{R}}^{-\frac{1}{2}} W_{i}, V_{i j}, \Lambda_{i} \pi_{W_{i}} S_{\mathcal{R}}^{-\frac{1}{2}}, v_{i j}\right)\right\}_{i \in \mathbb{\amalg}, j \in \mathbb{J}_{i}}$ is a Parseval RFF for $H$.

Example 3.16 We introduce atomic resolutions of an operator $R$ on $H$. Let $\mathcal{R}$ be an RFF for H. Suppose that $\widehat{\mathcal{R}}$ is the canonical dual RFF of $\mathcal{R}$. Then from Theorem 3.13, we have for all $f \in H$

$$
f=\sum_{i \in \mathbb{I}} \sum_{j \in \mathbb{J}_{i}} v_{i j}^{2} \pi_{W_{i}} \Lambda_{i}^{*} \tau_{V_{i j}} \widehat{\Lambda}_{i} \pi_{\widehat{W}_{i}}(f)=\sum_{i \in \mathbb{I}} \sum_{j \in \mathbb{J}_{i}} v_{i j}^{2} \pi_{\widehat{W}_{i}} \widehat{\Lambda}_{i}^{*} \tau_{V_{i j}} \Lambda_{i} \pi_{W_{i}}(f)
$$

This implies that

$$
I_{H}=\sum_{i \in \mathbb{I}} \sum_{j \in \mathbb{J}_{i}} v_{i j}^{2} \pi_{W_{i}} \Lambda_{i}^{*} \tau_{V_{i j}} \widehat{\Lambda}_{i} \pi_{\widehat{W}_{i}}=\sum_{i \in \mathbb{I}} \sum_{j \in \mathbb{J}_{i}} v_{i j}^{2} \pi_{\widehat{W}_{i}} \widehat{\Lambda}_{i}^{*} \tau_{V_{i j}} \Lambda_{i} \pi W_{i},
$$

and the series are convergent in the weak* sense. Let $R \in \mathcal{B}(H)$. As can be seen from the discussion above

$$
\begin{aligned}
R & =\sum_{i \in \mathbb{I}} \sum_{j \in \mathbb{J}_{i}} v_{i j}^{2} \pi_{W_{i}} \Lambda_{i}^{*} \tau_{V_{i j}} \widehat{\Lambda}_{i} \pi_{\widehat{W}_{i}} R=\sum_{i \in \mathbb{I}} \sum_{j \in \mathbb{J}_{i}} v_{i j}^{2} \pi_{\widehat{W}_{i}} \widehat{\Lambda}_{i}^{*} \tau_{V_{i j}} \Lambda_{i} \pi_{W_{i}} R \\
& =\sum_{i \in \mathbb{I}} \sum_{j \in \mathbb{J}_{i}} v_{i j}^{2} R \pi_{W_{i}} \Lambda_{i}^{*} \tau_{V_{i j}} \widehat{\Lambda}_{i} \pi_{\widehat{W}_{i}}=\sum_{i \in \mathbb{I}} \sum_{j \in \mathbb{J}_{i}} v_{i j}^{2} R \pi_{\widehat{W}_{i}} \widehat{\Lambda}_{i}^{*} \tau_{V_{i j}} \Lambda_{i} \pi_{W_{i}} .
\end{aligned}
$$




\subsection{Q-dual relay fusion frames}

The concept of Q-dual fusion frames for finite-dimensional Hilbert spaces and any separable Hilbert spaces were introduced in [13, 14], respectively. In this subsection we transfer some definitions and results of Q-dual fusion frames to the situation of RFFs. For more information about Q-dual fusion frames, we refer to [13, 14].

Throughout this subsection, the symbols $\mathcal{K}_{V}, \mathcal{K}_{U}, \mathcal{R}_{V}, \mathcal{R}_{U}$, and $\mathcal{L}_{T_{\mathcal{R}_{V}}}$ refer, respectively, to the spaces $\left(\sum_{i \in \mathbb{I}} \sum_{j \in \mathbb{J}_{i}} \oplus V_{i j}\right)_{\ell^{2}},\left(\sum_{i \in \mathbb{I}} \sum_{j \in \mathbb{J}_{i}} \oplus U_{i j}\right)_{\ell^{2}}$, the families $\left\{\left(W_{i}, V_{i j}, \Lambda_{i}, v_{i j}\right)\right\}_{i \in \mathbb{I}, j \in \mathbb{J}_{i}}$, $\left\{\left(M_{i}, U_{i j}, \Gamma_{i}, u_{i j}\right)\right\}_{i \in \mathbb{I}, j \in \mathbb{J}_{i}}$ and the collection of bounded left inverses of $T_{\mathcal{R}_{V}}$.

In analogy with the fusion frame case (see [14], Definition 3.1, 3.3), we introduce the following terminology.

Definition 9 Let $\mathcal{R}_{V}$ and $\mathcal{R}_{U}$ be two RFFs for $\mathrm{H}$. If there exists $Q \in \mathcal{B}\left(\mathcal{K}_{V}, \mathcal{K}_{U}\right)$ such that

$$
T_{\mathcal{R}_{U}}^{*} Q T_{\mathcal{R}_{V}}=I_{H}
$$

then $\mathcal{R}_{U}$ is said to be a Q-dual RFF of $\mathcal{R}_{V}$.

Definition 10 Let $P_{(m, n)}: \mathcal{K}_{V} \mapsto \mathcal{K}_{U}, P_{(m, n)}\left\{f_{i j}\right\}_{i \in \mathbb{I}, j \in \mathbb{J}_{i}}=\left\{\delta_{\{(m, n),(i, j)} f_{i j}\right\}_{i \in \mathbb{I}, j \in \mathbb{J}_{i}}$, where $\delta_{\{(m, n),(i, j)\}}$ is the Kronecker delta. If $\mathrm{Q}$ in Definition 9 satisfies

$$
Q P_{(m, n)} \mathcal{K}_{V}=P_{(m, n)} \mathcal{K}_{U}
$$

we say that $\mathrm{Q}$ is component preserving and $\mathcal{R}_{U}$ is a component preserving dual RFF of $\mathcal{R}_{V}$.

To simplify the exposition, we just formulate the following results which are analogous to Lemma 3.4, 3.5 of [14] with the proofs carrying over with small changes, so we omit them.

Lemma 3.17 Let $\mathcal{R}_{V}$ be an RFF for $H$. If $\mathcal{R}_{U}$ is a component preserving dual RFF of $\mathcal{R}_{V}$, then $U_{(m, n)}=A P_{(m, n)} \mathcal{K}_{V}$, for each $i \in \mathbb{I}, j \in \mathbb{J}_{i}$, where $A \in \mathcal{L}_{T_{\mathcal{R}_{V}}}$.

Theorem 3.18 Let $\mathcal{R}_{V}$ be an RFF for $H, A \in \mathcal{L}_{T_{\mathcal{R}_{V}}}$ and $U_{(m, n)}=A P_{(m, n)} \mathcal{K}_{V}$, for each $i \in$ $\mathbb{I}, j \in \mathbb{J}_{i}$. If $\mathcal{R}_{U}$ is a Bessel $r$-fusion sequence and

$$
Q_{A}: \mathcal{K}_{V} \mapsto \mathcal{K}_{U}, Q_{A}\left\{f_{i j}\right\}_{i \in \mathbb{I}, j \in \mathbb{J}_{i}}=\left\{\frac{1}{u_{i j}} A P_{(m, n)}\left\{f_{i j}\right\}_{i \in \mathbb{I}, j \in \mathbb{J}_{i}}\right\}_{m \in \mathbb{I}, n \in \mathbb{J}_{i}}
$$

is a well-defined bounded operator, then $\mathcal{R}_{U}$ is a $Q_{A}$-component preserving the dual $R F F$ of $\mathcal{R}_{V}$.

Remark 3.19 As discussed in [14], Remark 3.6, 3.10, we can always find the conditions for $\mathcal{R}_{U}$ being a Bessel r-fusion sequence and for $Q_{A}$ being a well defined bounded operator. In particular, the hypotheses $\mathcal{R}_{U}$ to be a Bessel r-fusion sequence and $Q_{A}$ to be a well-defined bounded operator cannot be avoided. 


\section{Relay fusion frame systems}

We begin this section by defining relay fusion frame systems. The use of RFFs permit furthermore local processing in each of the subspaces of relay spaces. For this purpose, it is useful to have a set of relay local frames for its subspaces.

Definition 11 Let $\mathcal{R}$ be a r-fusion frame for $\mathrm{H}$, and let $\mathcal{F}_{i j}=\left\{f_{i j k}\right\}_{k \in \mathbb{K}_{i j}}$ be a frame for $V_{i j}$ for each $i \in \mathbb{I}, j \in \mathbb{J}_{i}$. Then we call $\mathcal{F}_{i j}$ the $\mathrm{r}$-local frame with respect to $\mathrm{H}$ and $\left\{\mathcal{R}, \mathcal{F}=\left\{\mathcal{F}_{i j}\right\}\right\}$ a $\mathrm{r}$-fusion frame system for H. $\alpha$ and $\beta$ are the associated $\mathrm{r}$-fusion frame bounds if they are the fusion frame bounds for $\mathcal{R}$, and $\lambda$ and $\mu$ are the r-local frame bounds if these are the common frame bounds for the r-local frame $\mathcal{F}_{i j}$. A collection of dual frames $\widetilde{\mathcal{F}}_{i j}=\left\{\widetilde{f}_{i j k}\right\}_{k \in \mathbb{K}}$ for each $i \in \mathbb{I}, j \in \mathbb{J}_{i}$ associated with the r-local frames will be called r-local dual frames.

The next theorem generalizes a result of Cazassa and Kutyniok [4] to the situation of RFFs.

Theorem 4.1 Let $\left\{f_{i j k}\right\}_{k \in \mathbb{K}_{i j}}$ be a r-local frame with frame bounds $\lambda_{k}$ and $\mu_{k}$ and let $\left\{e_{i j k}\right\}_{k \in \mathbb{K}_{i j}}$ be an orthonormal basis for subspaces $V_{i j}$ for each $i \in \mathbb{I}, j \in \mathbb{J}_{i}$. Suppose that $0<\lambda=\inf _{k \in \mathbb{K}_{i j}} \lambda_{k} \leq \sup _{k \in \mathbb{K}_{i j}} \mu_{k}=\mu<+\infty$. Then the following statements are equivalent:

(i) $\mathcal{R}$ be an RFF for $H$.

(ii) $\left\{v_{i j} \pi_{W_{i}} \Lambda_{i}^{*} e_{i j k}\right\}_{i \in \mathbb{I}, j \in \mathbb{J}_{i}, k \in \mathbb{K}_{i j}}$ is a frame for $H$.

(iii) $\left\{v_{i j} \pi_{W_{i}} \Lambda_{i}^{*} f_{i j k}\right\}_{i \in \mathbb{I}, j \in \mathbb{J}_{i}, k \in \mathbb{K}_{i j}}$ is a frame for $H$.

In particular, if $\{\mathcal{R}, \mathcal{F}\}$ is an RFF system for $H$ with RFF bounds $\alpha$ and $\beta$, then $\left\{v_{i j} \pi_{W_{i}} \Lambda_{i}^{*} f_{i j k}\right\}_{i \in \mathbb{I}, j \in \mathbb{J}_{i}, k \in \mathbb{K}_{i j}}$ is a frame for $H$ with frame bounds $\alpha \lambda$ and $\beta \mu$. Also if $\left\{v_{i j} \pi_{W_{i}} \Lambda_{i}^{*} f_{i j k}\right\}_{i \in \mathbb{I}, j \in \mathbb{J}_{i}, k \in \mathbb{K}_{i j}}$ is a frame for $H$ with frame bounds $\alpha$ and $\beta$, then $\{\mathcal{R}, \mathcal{F}\}$ is an RFF system for $H$ with RFF bounds $\frac{\alpha}{\mu}$ and $\frac{\beta}{\lambda}$.

Proof (i) $\Leftrightarrow$ (ii) Note that

$$
\begin{aligned}
\sum_{i \in \mathbb{I}} \sum_{j \in \mathbb{J}_{i}} v_{i j}^{2}\left\|\tau_{V_{i j}} \Lambda_{i} \pi_{W_{i}}(f)\right\|^{2} & =\sum_{i \in \mathbb{I}} \sum_{j \in \mathbb{J}_{i}} v_{i j}^{2}\left\|\sum_{k \in \mathbb{K}_{i j}}\left\langle\Lambda_{i} \pi_{W_{i}}(f), e_{i j k}\right\rangle e_{i j k}\right\|^{2} \\
& =\sum_{i \in \mathbb{I}} \sum_{j \in \mathbb{J}_{i}} v_{i j}^{2} \sum_{k \in \mathbb{K}_{i j}}\left|\left\langle\Lambda_{i} \pi_{W_{i}}(f), e_{i j k}\right\rangle\right|^{2} \\
& =\sum_{i \in \mathbb{I}} \sum_{j \in \mathbb{J}_{i}} \sum_{k \in \mathbb{K}_{i j}}\left|\left\langle f, v_{i j} \pi_{W_{i}} \Lambda_{i}^{*}\left(e_{i j k}\right)\right\rangle\right|^{2}
\end{aligned}
$$

It follows that $\mathcal{R}$ is an RFF for $H$ if and only if $\left\{v_{i j} \pi_{W_{i}} \Lambda_{i}^{*} e_{i j k}\right\}_{i \in \mathbb{I}, j \in \mathbb{J}_{i}, k \in \mathbb{K}_{i j}}$ is a frame for $H$.

(i) $\Leftrightarrow$ (iii) By hypothesis, we obtain

$$
\begin{aligned}
\lambda \sum_{i \in \mathbb{I}} \sum_{j \in \mathbb{J}_{i}} v_{i j}^{2}\left\|\tau_{V_{i j}} \Lambda_{i} \pi_{W_{i}}(f)\right\|^{2} & \leq \sum_{i \in \mathbb{I}} \sum_{j \in \mathbb{J}_{i}} \lambda_{k} v_{i j}^{2}\left\|\tau_{V_{i j}} \Lambda_{i} \pi_{W_{i}}(f)\right\|^{2} \\
& \leq \sum_{i \in \mathbb{I}} \sum_{j \in \mathbb{J}_{i}} \sum_{k \in \mathbb{K}_{i j}} \|\left.\left\{f, v_{i j} \pi_{W_{i}} \Lambda_{i}^{*}\left(f_{i j k}\right)\right\rangle\right|^{2} \\
& \leq \mu \sum_{i \in \mathbb{I}} \sum_{j \in \mathbb{J}_{i}} v_{i j}^{2}\left\|\tau_{V_{i j}} \Lambda_{i} \pi_{W_{i}}(f)\right\|^{2} .
\end{aligned}
$$


If $\mathcal{R}$ is an RFF for $H$ with bounds $\alpha$ and $\beta$, then from the above calculations we have

$$
\alpha \lambda\|f\|^{2} \leq \sum_{i \in \mathbb{I}} \sum_{j \in \mathbb{J}_{i}} \sum_{k \in \mathbb{K}_{i j}}\left|\left\langle f, v_{i j} \pi_{W_{i}} \Lambda_{i}^{*}\left(f_{i j k}\right)\right\rangle\right|^{2} \leq \beta \mu\|f\|^{2} .
$$

Moreover, provided that $\left\{v_{i j} \pi_{W_{i}} \Lambda_{i}^{*} f_{i j k}\right\}_{i \in \mathbb{I}, j \in \mathbb{J}_{i}, k \in \mathbb{K}_{i j}}$ is a frame for $\mathrm{H}$ with frame bounds $\alpha$ and $\beta$, again by applying (3) we have

$$
\frac{\alpha}{\mu}\|f\|^{2} \leq \sum_{i \in \mathbb{I}} \sum_{j \in \mathbb{J}_{i}} v_{i j}^{2}\left\|\tau_{V_{i j}} \Lambda_{i} \pi_{W_{i}}(f)\right\|^{2} \leq \frac{\beta}{\lambda}\|f\|^{2}
$$

Corollary 4.2 Let $\left\{f_{i j k}\right\}_{k \in \mathbb{K}_{i j}}$ be a Parseval $r$-local frame and let $\left\{e_{i j k}\right\}_{k \in \mathbb{K}_{i j}}$ be an orthonormal basis for subspaces $V_{i j}$ for each $i \in \mathbb{I}, j \in \mathbb{J}_{i}$.. Then the following statements are equivalent:

(i) $\mathcal{R}$ is a Parseval RFF for $H$.

(ii) $S_{\mathcal{R}}=I_{H}$.

(iii) $\left\{v_{i j} \pi_{W_{i}} \Lambda_{i}^{*} e_{i j k}\right\}_{i \in \mathbb{I}, j \in \mathbb{J}_{i}, k \in \mathbb{K}_{i j}}$ is a Parseval frame for $H$.

(iv) $\left\{v_{i j} \pi_{W_{i}} \Lambda_{i}^{*} f_{i j k}\right\}_{i \in \mathbb{I}, j \in \mathbb{J}_{i}, k \in \mathbb{K}_{i j}}$ is a Parseval frame for $H$.

Proof By Theorem 4.1 it only remains to see (i) $\Leftrightarrow$ (ii). Applying Proposition 3.5, (i) implies (ii). Now suppose that $S_{\mathcal{R}}=I_{H}$. Then we have for all $f \in H$

$$
\begin{aligned}
\|f\|^{2} & =\left\langle S_{\mathcal{R}}(f), f\right\rangle=\left\langle\sum_{i \in \mathbb{I}} \sum_{j \in \mathbb{J}_{i}} v_{i j}^{2} \pi_{W_{i}} \Lambda_{i}^{*} \tau_{V_{i j}} \Lambda_{i} \pi_{W_{i}}(f), f\right\rangle \\
& =\sum_{i \in \mathbb{I}} \sum_{j \in \mathbb{J}_{i}} v_{i j}^{2}\left\|\tau_{V_{i j}} \Lambda_{i} \pi_{W_{i}}(f)\right\|^{2} .
\end{aligned}
$$

The following proposition will show the relation between the frame operator for $\mathcal{R}$ and the frame operator for the frame $\left\{v_{i j} \pi_{W_{i}} \Lambda_{i}^{*} f_{i j k}\right\}_{i \in \mathbb{\Pi}, j \in \mathbb{J}_{i}, k \in \mathbb{K}_{i j}}$.

Proposition 4.3 Let $\mathcal{R}$ be an RFF for $H$ and $\left\{f_{i j k}\right\}_{k \in \mathbb{K}_{i j}}$ be a Parseval $r$-local frame. Then the RFF operator $S_{\mathcal{R}}$ equals the frame operator $S_{v \pi \Lambda}$ for the frame $\left\{v_{i j} \pi_{W_{i}} \Lambda_{i}^{*} f_{i j k}\right\}_{i \in \mathbb{I}, j \in \mathbb{J}_{i}, k \in \mathbb{K}_{i j}}$, and, for all $h \in H$,

$$
\begin{aligned}
h & =\sum_{i \in \mathbb{I}} \sum_{j \in \mathbb{J}_{i}} v_{i j}^{2} S_{\mathcal{R}}^{-1} \pi_{W_{i}} \Lambda_{i}^{*} \tau_{V_{i j}} \Lambda_{i} \pi_{W_{i}}(h) \\
& =\sum_{i \in \mathbb{I}} \sum_{j \in \mathbb{J}_{i}} \sum_{k \in \mathbb{K}_{i j}}\left\langle h, v_{i j} \pi_{W_{i}} \Lambda_{i}^{*} f_{i j k}\right| S_{v \pi}^{-1} \nu_{i j} \pi_{W_{i}} \Lambda_{i}^{*} f_{i j k} .
\end{aligned}
$$

Proof Since $\left\{f_{i j k}\right\}_{k \in \mathbb{K}_{i j}}$ is a Parseval frame for $V_{i j}$ for each $i \in \mathbb{I}, j \in \mathbb{J}_{i}$,

$$
\tau_{V_{i j}} \Lambda_{i} \pi_{W_{i}}(h)=\sum_{k \in \mathbb{K}_{i j}}\left\langle\tau_{V_{i j}} \Lambda_{i} \pi_{W_{i}}(h), f_{i j k}\right\rangle f_{i j k}=\sum_{k \in \mathbb{K}_{i j}}\left\langle\Lambda_{i} \pi_{W_{i}}(h), f_{i j k}\right\rangle f_{i j k} .
$$

Therefore,

$$
S_{\mathcal{R}}(h)=\sum_{i \in \mathbb{I}} \sum_{j \in \mathbb{J}_{i}} v_{i j}^{2} \pi_{W_{i}} \Lambda_{i}^{*} \tau_{V_{i j}} \Lambda_{i} \pi_{W_{i}}(h)
$$




$$
\begin{aligned}
& =\sum_{i \in \mathbb{I}} \sum_{j \in \mathbb{J}_{i}} v_{i j}^{2} \pi_{W_{i}} \Lambda_{i}^{*} \sum_{k \in \mathbb{K}_{i j}}\left\langle\Lambda_{i} \pi_{W_{i}}(h), f_{i j k}\right\rangle f_{i j k} \\
& =\sum_{i \in \mathbb{I}} \sum_{j \in \mathbb{J}_{i}} \sum_{k \in \mathbb{K}_{i j}}\left\langle h, v_{i j} \pi_{W_{i}} \Lambda_{i}^{*} f_{i j k}\right\rangle v_{i j} \pi_{W_{i}} \Lambda_{i}^{*} f_{i j k} \\
& =S_{v \pi \Lambda}(h)
\end{aligned}
$$

and

$$
\begin{aligned}
h & =\sum_{i \in \mathbb{I}} \sum_{j \in \mathbb{J}_{i}} v_{i j}^{2} S_{\mathcal{R}}^{-1} \pi_{W_{i}} \Lambda_{i}^{*} \tau_{V_{i j}} \Lambda_{i} \pi_{W_{i}}(h) \\
& =\sum_{i \in \mathbb{I}} \sum_{j \in \mathbb{J}_{i}} v_{i j}^{2} S_{\mathcal{R}}^{-1} \pi_{W_{i}} \Lambda_{i}^{*} \sum_{k \in \mathbb{K}_{i j}}\left\langle\Lambda_{i} \pi_{W_{i}}(h), f_{i j k}\right) f_{i j k} \\
& =\sum_{i \in \mathbb{I}} \sum_{j \in \mathbb{J}_{i}} \sum_{k \in \mathbb{K}_{i j}}\left\langle h, v_{i j} \pi_{W_{i}} \Lambda_{i}^{*} f_{i j k}\right| S_{v \pi \Lambda}^{-1} v_{i j} \pi_{W_{i}} \Lambda_{i}^{*} f_{i j k} .
\end{aligned}
$$

The following result gives another representation of the frame operator for $\mathcal{R}$.

Proposition 4.4 Let $\{\mathcal{R}, \mathcal{F}\}$ be an RFF system for $H$, and let $\widetilde{\mathcal{F}}_{i j}=\left\{\widetilde{f}_{i j k}\right\}_{k \in \mathbb{K}_{i j}}$ be associated $r$-local dual frames. Then the associated RFF operator $S_{\mathcal{R}}$ can be written as

$$
S_{\mathcal{R}}=\sum_{i \in \mathbb{I}} \sum_{j \in \mathbb{J}_{i}} v_{i j}^{2} \pi_{W_{i}} \Lambda_{i}^{*} T_{\widetilde{\mathcal{F}}_{i j}}^{*} T_{\mathcal{F}_{i j}} \Lambda_{i} \pi_{W_{i}}=\sum_{i \in \mathbb{I}} \sum_{j \in \mathbb{J}_{i}} v_{i j}^{2} \pi_{W_{i}} \Lambda_{i}^{*} T_{\mathcal{F}_{i j}}^{*} T_{\widetilde{\mathcal{F}}_{i j}} \Lambda_{i} \pi_{W_{i}}
$$

Proof For each $f \in H$, we have

$$
\begin{aligned}
S_{\mathcal{R}}(f) & =\sum_{i \in \mathbb{I}} \sum_{j \in \mathbb{J}_{i}} v_{i j}^{2} \pi_{W_{i}} \Lambda_{i}^{*} \tau_{V_{i j}} \Lambda_{i} \pi_{W_{i}}(f) \\
& =\sum_{i \in \mathbb{I}} \sum_{j \in \mathbb{J}_{i}} v_{i j}^{2} \pi_{W_{i}} \Lambda_{i}^{*} \sum_{k \in \mathbb{K}_{i j}}\left\langle\Lambda_{i} \pi_{W_{i}}(f), f_{i j k} \widetilde{f}_{i j k}\right. \\
& =\sum_{i \in \mathbb{I}} \sum_{j \in \mathbb{J}_{i}} v_{i j}^{2} \pi_{W_{i}} \Lambda_{i}^{*} T_{\widetilde{\mathcal{F}}_{i j}}^{*} T_{\mathcal{F}_{i j}} \Lambda_{i} \pi_{W_{i}}(f) .
\end{aligned}
$$

Similarly,

$$
\begin{aligned}
S_{\mathcal{R}}(f) & =\sum_{i \in \mathbb{I}} \sum_{j \in \mathbb{J}_{i}} v_{i j}^{2} \pi_{W_{i}} \Lambda_{i}^{*} \tau_{V_{i j}} \Lambda_{i} \pi_{W_{i}}(f) \\
& =\sum_{i \in \mathbb{I}} \sum_{j \in \mathbb{J}_{i}} v_{i j}^{2} \pi_{W_{i}} \Lambda_{i}^{*} \sum_{k \in \mathbb{K}_{i j}}\left\langle\Lambda_{i} \pi_{W_{i}}(f), \widetilde{f}_{i j k}\right\rangle f_{i j k} \\
& =\sum_{i \in \mathbb{I}} \sum_{j \in \mathbb{J}_{i}} v_{i j}^{2} \pi_{W_{i}} \Lambda_{i}^{*} T_{\mathcal{F}_{i j}}^{*} T_{\widetilde{\mathcal{F}}_{i j}} \Lambda_{i} \pi_{W_{i}}(f) .
\end{aligned}
$$

The following theorem will provide accurate estimates for the RFF operators of an RFF of the form $\left\{\left(A W_{i}, V_{i j}, \Lambda_{i}, v_{i j}\right)\right\}_{i \in \mathbb{I}, j \in \mathbb{J}_{i}}$.

Theorem 4.5 Let $\mathcal{R}=\left\{\left(W_{i}, V_{i j}, \Lambda_{i}, v_{i j}\right)\right\}_{i \in \mathbb{I}, j \in \mathbb{J}_{i}}$ be an RFF for $H$ with RFF operator $S_{\mathcal{R}}$ and let $A \in \mathcal{B}(H)$ be an invertible operator. Then $\mathcal{R}_{\mathcal{A}}=\left\{\left(A W_{i}, V_{i j}, \Lambda_{i}, v_{i j}\right)\right\}_{i \in \mathbb{I}, j \in \mathbb{J}_{i}}$ is an RFF for $H$ 
with RFF operator $S_{\mathcal{R}_{\mathcal{A}}}$ satisfying

$$
\frac{A S_{\mathcal{R}} A^{*}}{\|A\|^{2}} \leq S_{\mathcal{R}_{\mathcal{A}}} \leq\left\|A^{-1}\right\|^{2} A S_{\mathcal{R}} A^{*}
$$

Proof For all $f \in H$, we have

$$
\begin{aligned}
\left\langle\frac{A S_{\mathcal{R}} A^{*}}{\|A\|^{2}} f, f\right\rangle & =\frac{1}{\|A\|^{2}} \sum_{i \in \mathbb{I}} \sum_{j \in \mathbb{J}_{i}} v_{i j}^{2}\left\|\tau_{V_{i j}} \Lambda_{i} \pi_{W_{i}} A^{*}(f)\right\|^{2} \\
& =\frac{1}{\|A\|^{2}} \sum_{i \in \mathbb{I}} \sum_{j \in \mathbb{J}_{i}} v_{i j}^{2}\left\|\tau_{V_{i j}} \Lambda_{i} \pi_{W_{i}} A^{*} \pi_{A W_{i}}(f)\right\|^{2} \\
& \leq \frac{\left\|A^{*}\right\|^{2}}{\|A\|^{2}} \sum_{i \in \mathbb{I}} \sum_{j \in \mathbb{J}_{i}} v_{i j}^{2}\left\|\tau_{V_{i j}} \Lambda_{i} \pi_{A W_{i}}(f)\right\|^{2} \\
& =\left\langle S_{\mathcal{R}_{\mathcal{A}}} f, f\right\rangle .
\end{aligned}
$$

Applying Lemma 3.8 to $A^{-1}$ and $A W_{i}$ yields

$$
\pi_{A W_{i}}=\pi_{A W_{i}}\left(A^{-1}\right)^{*} \pi_{W_{i}} A^{*}
$$

Then

$$
\begin{aligned}
\left\langle S_{\left.\mathcal{R}_{\mathcal{A}} f, f\right\rangle}\right. & =\sum_{i \in \mathbb{I}} \sum_{j \in \mathbb{J}_{i}} v_{i j}^{2}\left\|\tau_{V_{i j}} \Lambda_{i} \pi_{A W_{i}}(f)\right\|^{2} \\
& =\sum_{i \in \mathbb{I}} \sum_{j \in \mathbb{J}_{i}} v_{i j}^{2}\left\|\tau_{V_{i j}} \Lambda_{i} \pi_{A W_{i}}\left(A^{-1}\right)^{*} \pi_{W_{i}} A^{*}(f)\right\|^{2} \\
& \leq\left\|\left(A^{-1}\right)^{*}\right\|^{2} \sum_{i \in \mathbb{I}} \sum_{j \in \mathbb{J}_{i}} v_{i j}{ }_{i j}\left\|\tau_{V_{i j}} \Lambda_{i} \pi_{W_{i}} A^{*}(f)\right\|^{2} \\
& =\left\|\left(A^{-1}\right)^{*}\right\|^{2}\left\langle S_{\mathcal{R}_{\mathcal{A}}} A^{*} f, A^{*} f\right\rangle \\
& =\left\langle\left\|\left(A^{-1}\right)^{*}\right\|^{2} A S_{\mathcal{R}_{\mathcal{A}}} A^{*} f, f\right\rangle .
\end{aligned}
$$

The following result can be regarded as a corollary of Theorem 4.5. Here we show it in a different way.

Theorem 4.6 Let $\mathcal{R}=\left\{\left(W_{i}, V_{i j}, \Lambda_{i}, v_{i j}\right)\right\}_{i \in \mathbb{I}, j \in \mathbb{J}_{i}}$ be an RFF for $H$ with RFF bounds $\alpha$ and $\beta$. If $A \in \mathcal{B}(H)$ is an invertible operator, then $\mathcal{R}_{\mathcal{A}}=\left\{\left(A W_{i}, V_{i j}, \Lambda_{i}, v_{i j}\right)\right\}_{i \in \mathbb{I}, j \in \mathbb{J}_{i}}$ is an RFF for $H$ with RFF bounds

$$
\frac{\alpha}{\left\|A^{*}\right\|^{2}\left\|\left(A^{*}\right)^{-1}\right\|^{2}}, \quad \beta\left\|A^{*}\right\|^{2}\left\|\left(A^{*}\right)^{-1}\right\|^{2}
$$

Proof By applying Lemma 3.8, we obtain

$$
\left\|\tau_{V_{i j}} \Lambda_{i} \pi_{W_{i}} A^{*}(f)\right\|=\left\|\tau_{V_{i j}} \Lambda_{i} \pi_{W_{i}} A^{*} \pi_{A W_{i}}(f)\right\| \leq\left\|A^{*}\right\|\left\|\tau_{V_{i j}} \Lambda_{i} \pi_{A W_{i}}(f)\right\| .
$$


Since $A^{*}(f) \in H$ and $\mathcal{R}$ is an RFF for $H$, we have

$$
\alpha\left\|A^{*}(f)\right\|^{2} \leq \sum_{i \in \mathbb{I}} \sum_{j \in \mathbb{J}_{i}} v_{i j}^{2}\left\|\tau_{V_{i j}} \Lambda_{i} \pi_{W_{i}} A^{*}(f)\right\|^{2} \leq\left\|A^{*}\right\|^{2} \sum_{i \in \mathbb{I}} \sum_{j \in \mathbb{J}_{i}} v_{i j}^{2}\left\|\tau_{V_{i j}} \Lambda_{i} \pi_{A W_{i}}(f)\right\|^{2} .
$$

Due to $A^{*}$ being an invertible operator, we have

$$
\frac{\alpha}{\left\|A^{*}\right\|^{2}\left\|\left(A^{*}\right)^{-1}\right\|^{2}}\|f\|^{2} \leq \sum_{i \in \mathbb{I}} \sum_{j \in \mathbb{J}_{i}} v_{i j}^{2}\left\|\tau_{V_{i j}} \Lambda_{i} \pi_{A W_{i}}(f)\right\|^{2}
$$

In order to show the upper bound, notice that applying Lemma 3.8 to $A^{-1}$ and $A W_{i}$ yields

$$
\pi_{A W_{i}}=\pi_{A W_{i}}\left(A^{-1}\right)^{*} \pi_{W_{i}} A^{*}
$$

Therefore

$$
\left\|\tau_{V_{i j}} \Lambda_{i} \pi_{A W_{i}}(f)\right\| \leq\left\|\left(A^{-1}\right)^{*}\right\|\left\|\tau_{V_{i j}} \Lambda_{i} \pi_{W_{i}} A^{*}(f)\right\|
$$

Hence

$$
\begin{aligned}
\sum_{i \in \mathbb{I}} \sum_{j \in \mathbb{J}_{i}} v_{i j}^{2}\left\|\tau_{V_{i j}} \Lambda_{i} \pi_{A W_{i}}(f)\right\|^{2} & \leq\left\|\left(A^{-1}\right)^{*}\right\|^{2} \sum_{i \in \mathbb{I}} \sum_{j \in \mathbb{J}_{i}} v_{i j}^{2}\left\|\tau_{V_{i j}} \Lambda_{i} \pi_{W_{i}} A^{*}(f)\right\|^{2} \\
& \leq \beta\left\|A^{*}\right\|^{2}\left\|\left(A^{*}\right)^{-1}\right\|^{2}\|f\|^{2} .
\end{aligned}
$$

Similarly, we have the following.

Theorem 4.7 Let $\mathcal{R}=\left\{\left(W_{i}, V_{i j}, \Lambda_{i}, v_{i j}\right)\right\}_{i \in \mathbb{I}, j \in \mathbb{J}_{i}}$ be an RFF for $H$ with RFF bounds $\alpha$ and $\beta$. If $B_{i} \in \mathcal{B}\left(K_{i}\right)$ are invertible operators for each $i \in \mathbb{I}$, then $\mathcal{R}_{\mathcal{B}}=\left\{\left(W_{i}, B_{i} V_{i j}, \Lambda_{i}, v_{i j}\right)\right\}_{i \in \mathbb{I}, j \in \mathbb{J}_{i}}$ is an RFF for $H$ with RFF bounds

$$
\min _{i \in \mathbb{I}}\left\{\frac{\alpha}{\left\|B_{i}\right\|^{2}\left\|B_{i}^{-1}\right\|^{2}}\right\}, \quad \max _{i \in \mathbb{I}}\left\{\left\|B_{i}^{*}\right\|^{2}\left\|\left(B_{i}^{-1}\right)^{*}\right\|^{2} \beta\right\}
$$

Proof For any $f \in H$, by Lemma 3.8 we have

$$
\left\|B_{i} \tau_{V_{i j}} \Lambda_{i} \pi_{W_{i}}(f)\right\|=\left\|\tau_{B_{i} V_{i j}} B_{i} \tau_{V_{i j}} \Lambda_{i} \pi_{W_{i}}(f)\right\| \leq\left\|B_{i}\right\|\left\|\tau_{B_{i} V_{i j}} \Lambda_{i} \pi_{W_{i}}(f)\right\| .
$$

Then

$$
\frac{1}{\left\|B_{i}\right\|\left\|B_{i}^{-1}\right\|}\left\|\tau_{V_{i j}} \Lambda_{i} \pi_{W_{i}}(f)\right\| \leq\left\|\tau_{B_{i} V_{i j}} \Lambda_{i} \pi_{W_{i}}(f)\right\|
$$

It follows that

$$
\begin{aligned}
\min _{i \in \mathbb{I}}\left\{\frac{\alpha}{\left\|B_{i}\right\|^{2}\left\|B_{i}^{-1}\right\|^{2}}\right\}\|f\|^{2} & \leq \sum_{i \in \mathbb{I}} \sum_{j \in \mathbb{J}_{i}} \frac{v_{i j}^{2}}{\left\|B_{i}\right\|^{2}\left\|B_{i}^{-1}\right\|^{2}}\left\|\tau_{V_{i j}} \Lambda_{i} \pi_{W_{i}}(f)\right\|^{2} \\
& \leq \sum_{i \in \mathbb{I}} \sum_{j \in \mathbb{J}_{i}} v_{i j}^{2}\left\|\tau_{B_{i} V_{i j}} \Lambda_{i} \pi_{W_{i}}(f)\right\|^{2} .
\end{aligned}
$$


As in Theorem 4.6, we have

$$
\tau_{B_{i} V_{i j}}=\tau_{B_{i} V_{i j}}\left(B_{i}^{-1}\right)^{*} \tau_{V_{i j}} B_{i}^{*} .
$$

Thus

$$
\begin{aligned}
\left\|\tau_{B_{i} V_{i j}} \Lambda_{i} \pi_{W_{i}}(f)\right\| & =\left\|\tau_{B_{i} V_{i j}}\left(B_{i}^{-1}\right)^{*} \tau_{V_{i j}} B_{i}^{*} \Lambda_{i} \pi_{W_{i}}(f)\right\| \\
& \leq\left\|B_{i}^{*}\right\|\left\|\left(B_{i}^{-1}\right)^{*}\right\|\left\|\tau_{V_{i j}} \Lambda_{i} \pi_{W_{i}}(f)\right\| .
\end{aligned}
$$

Hence

$$
\begin{aligned}
\sum_{i \in \mathbb{I}} \sum_{j \in \mathbb{J}_{i}} v_{i j}^{2}\left\|\tau_{B_{i} V_{i j}} \Lambda_{i} \pi_{W_{i}}(f)\right\|^{2} & \leq \sum_{i \in \mathbb{I}} \sum_{j \in \mathbb{J}_{i}}\left\|B_{i}^{*}\right\|^{2}\left\|\left(B_{i}^{-1}\right)^{*}\right\|^{2} v_{i j}^{2}\left\|\tau_{V_{i j}} \Lambda_{i} \pi_{W_{i}}(f)\right\|^{2} \\
& \leq \max _{i \in \mathbb{I}}\left\{\left\|B_{i}^{*}\right\|^{2}\left\|\left(B_{i}^{-1}\right)^{*}\right\|^{2} \beta\right\}\|f\|^{2} .
\end{aligned}
$$

Specializing to $A$ and $\left\{B_{i}\right\}_{i \in \mathbb{I}}$ being the inverse RFF operator $S_{\mathcal{R}}^{-1}$ and the inverse local relay frame operators $\left\{S_{i}^{-1}\right\}_{i \in \mathbb{I}}$, we obtain from Theorems 4.6 and 4.7 Corollaries 4.8 and 4.9 , respectively.

Corollary 4.8 Let $\mathcal{R}=\left\{\left(W_{i}, V_{i j}, \Lambda_{i}, v_{i j}\right)\right\}_{i \in \mathbb{I}, j \in \mathbb{J}_{i}}$ be an RFF for $H$ with RFF bounds $\alpha$ and $\beta$ and RFF operator $S_{\mathcal{R}}$. Then $\left\{\left(S_{\mathcal{R}}^{-1} W_{i}, V_{i j}, \Lambda_{i}, v_{i j}\right)\right\}_{i \in \mathbb{I}, j \in \mathbb{J}}$ is an RFF for H with RFF bounds $\frac{\alpha^{2}}{\beta}$ and $\frac{\beta^{2}}{\alpha}$.

Corollary 4.9 Let $\mathcal{R}=\left\{\left(W_{i}, V_{i j}, \Lambda_{i}, v_{i j}\right)\right\}_{i \in \mathbb{I}, j \in \mathbb{J}_{i}}$ be an RFF for $H$ with RFF bounds $\alpha$ and $\beta$ and relay local frame operator $S_{i}$. Then $\left\{\left(W_{i}, S_{i}^{-1} V_{i j}, \Lambda_{i}, v_{i j}\right)\right\}_{i \in \mathbb{I}, j \in \mathbb{J}_{i}}$ is an RFF for $H$ with RFF bounds

$$
\min _{i \in \mathbb{I}}\left\{\frac{\alpha}{\left\|S_{i}\right\|^{2}\left\|S_{i}^{-1}\right\|^{2}}\right\}, \quad \max _{i \in \mathbb{I}}\left\{\left\|S_{i}^{*}\right\|^{2}\left\|\left(S_{i}^{-1}\right)^{*}\right\|^{2} \beta\right\} .
$$

The following results show that $\left\{S_{\mathcal{R}}^{-1} v_{i j} \pi_{W_{i}} \Lambda_{i}^{*} \tilde{f}_{i j k}\right\}_{i \in \mathbb{I}, j \in \mathbb{J}_{i}, k \in \mathbb{K}_{i j}}$ is a dual frame for the frame $\left\{v_{i j} \pi_{W_{i}} \Lambda_{i}^{*} f_{i j k}\right\}_{i \in \mathbb{I}, j \in \mathbb{J}_{i}, k \in \mathbb{K}_{i j}}$ and a "dual" relation also holds.

Proposition 4.10 Let $\{\mathcal{R}, \mathcal{F}\}$ be an RFF system for $H$ with associated RFF operator $S_{\mathcal{R}}$, common relay local frame bounds and relay local dual frames $\left\{\tilde{f}_{i j k}\right\}_{k \in \mathbb{K}_{i j}}, i \in \mathbb{I}, j \in \mathbb{J}_{i}$. Then $\left\{S_{\mathcal{R}}^{-1} v_{i j} \pi_{W_{i}} \Lambda_{i}^{*} \tilde{f}_{i j k}\right\}_{i \in \mathbb{I}, j \in \mathbb{J}, k \in \mathbb{K}_{i j}}$ is a dual frame for the frame $\left\{v_{i j} \pi_{W_{i}} \Lambda_{i}^{*} f_{i j k}\right\}_{i \in \mathbb{I}, j \in \mathbb{J}_{i}, k \in \mathbb{K}_{i j}}$.

Proof For all $f \in H$, we have

$$
\begin{aligned}
& \sum_{i \in \mathbb{I}} \sum_{j \in \mathbb{J}_{i}} \sum_{k \in \mathbb{K}_{i j}}\left\langle f, S_{\mathcal{R}}^{-1} v_{i j} \pi_{W_{i}} \Lambda_{i}^{*} \tilde{f}_{i j k}\right\rangle v_{i j} \pi_{W_{i}} \Lambda_{i}^{*} f_{i j k} \\
& \quad=\sum_{i \in \mathbb{I}} \sum_{j \in \mathbb{J}_{i}} v_{i j}^{2} \pi_{W_{i}} \Lambda_{i}^{*} \sum_{k \in \mathbb{K}_{i j}}\left\langle\tau_{V_{i j}} \Lambda_{i} \pi_{W_{i}} S_{\mathcal{R}}^{-1}(f), \tilde{f}_{i j k}\right\rangle f_{i j k} \\
& \quad=\sum_{i \in \mathbb{I}} \sum_{j \in \mathbb{J}_{i}} v_{i j}^{2} \pi_{W_{i}} \Lambda_{i}^{*} \tau_{V_{i j}} \Lambda_{i} \pi_{W_{i}} S_{\mathcal{R}}^{-1}(f) \\
& \quad=f .
\end{aligned}
$$


Proposition 4.11 Let $\{\mathcal{R}, \mathcal{F}\}$ be an RFF system for $H$ with associated RFF operator $S_{\mathcal{R}}$, common relay local frame bounds and relay local dual frames $\left\{\tilde{f}_{i j k}\right\}_{k \in \mathbb{K}_{i j}}, i \in \mathbb{I}, j \in \mathbb{J}_{i}$. Then $\left\{v_{i j} \pi_{W_{i}} \Lambda_{i}^{*} \tilde{f}_{i j k}\right\}_{i \in \mathbb{I}, j \in \mathbb{J}_{i}, k \in \mathbb{K}_{i j}}$ is a dual frame for the frame $\left\{S_{\mathcal{R}}^{-1} v_{i j} \pi_{W_{i}} \Lambda_{i}^{*} f_{i j k}\right\}_{i \in \mathbb{I}, j \in \mathbb{J}_{i}, k \in \mathbb{K}_{i j}}$.

Proof Employing Proposition 4.10, we have for all $f \in H$

$$
\begin{aligned}
f & =\sum_{i \in \mathbb{I}} \sum_{j \in \mathbb{J}_{i}} \sum_{k \in \mathbb{K}_{i j}}\left\langle f, v_{i j} \pi_{W_{i}} \Lambda_{i}^{*} f_{i j k}\right\rangle S_{\mathcal{R}}^{-1} v_{i j} \pi_{W_{i}} \Lambda_{i}^{*} \tilde{f}_{i j k} \\
& =S_{\mathcal{R}}^{-1} \sum_{i \in \mathbb{I}} \sum_{j \in \mathbb{J}_{i}} v_{i j}^{2} \pi_{W_{i}} \Lambda_{i}^{*} \sum_{k \in \mathbb{K}_{i j}}\left\langle\tau_{V_{i j}} \Lambda_{i} \pi_{W_{i}}(f), \tilde{f}_{i j k}\right\rangle f_{i j k} \\
& =\sum_{i \in \mathbb{I}} \sum_{j \in \mathbb{J}_{i}} \sum_{k \in \mathbb{K}_{i j}}\left\langle f, v_{i j} \pi_{W_{i}} \Lambda_{i}^{*} \tilde{f}_{i j k}\right\rangle S_{\mathcal{R}}^{-1} v_{i j} \pi_{W_{i}} \Lambda_{i}^{*} f_{i j k} .
\end{aligned}
$$

\section{Perturbation of the relay fusion frames}

The stability of frames is of great significance in practice, so many authors have carried out extensive research on it, e.g., see [3, 6, 7, 22, 24]. In this section, we study the stability of RFFs. Similar to ordinary frames, RFFs are stable under small perturbations. Specifically, we have the following.

Theorem 5.1 Let $\mathcal{R}_{1}=\left\{\left(W_{i}, V_{i j}, \Lambda_{i}, v_{i j}\right)\right\}_{i \in \mathbb{I}, j \in \mathbb{J}_{i}}$ be an RFF for $H$ with RFF bounds $\alpha$ and $\beta$. Suppose that $\left\{Z_{i j}\right\}_{j \in \mathbb{J}_{i}}$ is a family of closed subspaces in $K_{i}$ for each $i \in \mathbb{I}$ and there exist constants $\delta_{1}, \delta_{2}, \varepsilon \geq 0$ such that $\max \left\{\delta_{1}+\frac{\varepsilon}{\sqrt{\alpha}}, \delta_{2}\right\}<1$ and for all $f \in H$

$$
\begin{aligned}
& \left(\sum_{i \in \mathbb{I}} \sum_{j \in \mathbb{J}_{i}} v_{i j}^{2}\left\|\tau_{V_{i j}} \Lambda_{i} \pi_{W_{i}}(f)-\tau_{Z_{i j}} \Lambda_{i} \pi_{W_{i}}(f)\right\|^{2}\right)^{\frac{1}{2}} \\
& \leq \delta_{1}\left(\sum_{i \in \mathbb{I}} \sum_{j \in \mathbb{J}_{i}} v_{i j}^{2}\left\|\tau_{V_{i j}} \Lambda_{i} \pi_{W_{i}}(f)\right\|^{2}\right)^{\frac{1}{2}} \\
& \quad+\delta_{2}\left(\sum_{i \in \mathbb{I}} \sum_{j \in \mathbb{J}_{i}} v_{i j}^{2}\left\|\tau_{Z_{i j}} \Lambda_{i} \pi_{W_{i}}(f)\right\|^{2}\right)^{\frac{1}{2}}+\varepsilon\|f\| .
\end{aligned}
$$

Then $\mathcal{R}_{2}=\left\{\left(W_{i}, Z_{i j}, \Lambda_{i}, v_{i j}\right)\right\}_{i \in \mathbb{\Pi}, j \in \mathbb{J}_{i}}$ is an RFF for $H$ with RFF bounds

$$
\alpha\left(\frac{1-\delta_{1}-\frac{\varepsilon}{\sqrt{\alpha}}}{1+\delta_{2}}\right)^{2}, \quad \beta\left(\frac{1+\delta_{1}+\frac{\varepsilon}{\sqrt{\beta}}}{1-\delta_{2}}\right)^{2} .
$$

Proof We first prove the lower bound. Since $\mathcal{R}_{1}$ is an RFF for $H$ with bounds $\alpha$ and $\beta$, then, for each $f \in H$, we obtain

$$
\frac{1}{\beta} \sum_{i \in \mathbb{I}} \sum_{j \in \mathbb{J}_{i}} v_{i j}^{2}\left\|\tau_{V_{i j}} \Lambda_{i} \pi_{W_{i}}(f)\right\|^{2} \leq\|f\|^{2} \leq \frac{1}{\alpha} \sum_{i \in \mathbb{I}} \sum_{j \in \mathbb{J}_{i}} v_{i j}^{2}\left\|\tau_{V_{i j}} \Lambda_{i} \pi_{W_{i}}(f)\right\|^{2} .
$$

By using (4) and the triangle inequality, we have

$$
\left(\sum_{i \in \mathbb{I}} \sum_{j \in \mathbb{J}_{i}} v_{i j}^{2}\left\|\tau_{V_{i j}} \Lambda_{i} \pi_{W_{i}}(f)\right\|^{2}\right)^{\frac{1}{2}}-\left(\sum_{i \in \mathbb{I}} \sum_{j \in \mathbb{J}_{i}} v_{i j}^{2}\left\|\tau_{Z_{i j}} \Lambda_{i} \pi_{W_{i}}(f)\right\|^{2}\right)^{\frac{1}{2}}
$$




$$
\begin{aligned}
& \leq\left(\sum_{i \in \mathbb{I}} \sum_{j \in \mathbb{J}_{i}} v_{i j}^{2}\left\|\tau_{V_{i j}} \Lambda_{i} \pi_{W_{i}}(f)-\tau_{Z_{i j}} \Lambda_{i} \pi_{W_{i}}(f)\right\|^{2}\right)^{\frac{1}{2}} \\
& \leq\left(\delta_{1}+\frac{\varepsilon}{\sqrt{\alpha}}\right)\left(\sum_{i \in \mathbb{I}} \sum_{j \in \mathbb{J}_{i}} v_{i j}^{2}\left\|\tau_{V_{i j}} \Lambda_{i} \pi_{W_{i}}(f)\right\|^{2}\right)^{\frac{1}{2}}+\delta_{2}\left(\sum_{i \in \mathbb{I}} \sum_{j \in \mathbb{J}_{i}} v_{i j}^{2}\left\|\tau_{Z_{i j}} \Lambda_{i} \pi_{W_{i}}(f)\right\|^{2}\right)^{\frac{1}{2}} .
\end{aligned}
$$

Now solving for $\sum_{i \in \mathbb{I}} \sum_{j \in \mathbb{J}_{i}} v_{i j}^{2}\left\|\tau_{Z_{i j}} \Lambda_{i} \pi_{W_{i}}(f)\right\|^{2}$ yields

$$
\alpha\left(\frac{1-\delta_{1}-\frac{\varepsilon}{\sqrt{\alpha}}}{1+\delta_{2}}\right)^{2}\|f\|^{2} \leq \sum_{i \in \mathbb{I}} \sum_{j \in \mathbb{J}_{i}} v_{i j}^{2}\left\|\tau_{Z_{i j}} \Lambda_{i} \pi_{W_{i}}(f)\right\|^{2} .
$$

Similarly we can prove that

$$
\beta\left(\frac{1+\delta_{1}+\frac{\varepsilon}{\sqrt{\beta}}}{1-\delta_{2}}\right)^{2}\|f\|^{2} \geq \sum_{i \in \mathbb{I}} \sum_{j \in \mathbb{J}_{i}} v_{i j}^{2}\left\|\tau_{Z_{i j}} \Lambda_{i} \pi_{W_{i}}(f)\right\|^{2} .
$$

The following is another version of perturbation of RFFs.

Theorem 5.2 Let $\mathcal{R}_{1}=\left\{\left(W_{i}, V_{i j}, \Lambda_{i}, v_{i j}\right)\right\}_{i \in \mathbb{I}, j \in \mathbb{\Xi}_{i}}$ be an RFF for $H$ with RFF bounds $\alpha$ and $\beta$. Suppose that $\left\{Z_{i j}\right\}_{j \in \mathbb{J}_{i}}$ is a family of closed subspaces in $K_{i}$ for each $i \in \mathbb{I}$ and there exists a constant $0<\delta<\alpha$ such that

$$
\sum_{i \in \mathbb{I}} \sum_{j \in \mathbb{J}_{i}} v_{i j}^{2}\left\|\tau_{V_{i j}} \Lambda_{i} \pi_{W_{i}}(f)-\tau_{Z_{i j}} \Lambda_{i} \pi_{W_{i}}(f)\right\|^{2} \leq \delta\|f\|^{2}, \quad \forall f \in H
$$

Then $\mathcal{R}_{2}=\left\{\left(W_{i}, Z_{i j}, \Lambda_{i}, v_{i j}\right)\right\}_{i \in \mathbb{I}, j \in \mathbb{J}_{i}}$ is an RFF for H with RFF bounds

$$
\sqrt{\delta}-\sqrt{\alpha}, \quad \sqrt{\delta}+\sqrt{\beta} .
$$

Proof By applying the triangle inequality, we obtain

$$
\begin{aligned}
& \left\|\left\{v_{i j} \tau_{Z_{i j}} \Lambda_{i} \pi_{W_{i}}(f)\right\}_{i \in \mathbb{I}, j \in \mathbb{J}_{i}}\right\| \\
& \quad \leq\left\|\left\{v_{i j} \tau_{V_{i j}} \Lambda_{i} \pi_{W_{i}}(f)-v_{i j} \tau_{Z_{i j}} \Lambda_{i} \pi_{W_{i}}(f)\right\}_{i \in \mathbb{I}, j \in \mathbb{J}_{i}}\right\|+\left\|\left\{v_{i j} \tau_{V_{i j}} \Lambda_{i} \pi_{W_{i}}(f)\right\}_{i \in \mathbb{I}, j \in \mathbb{J}_{i}}\right\| \\
& \leq(\sqrt{\delta}+\sqrt{\beta})\|f\|,
\end{aligned}
$$

for all $f \in H$. Therefore

$$
\sum_{i \in \mathbb{I}} \sum_{j \in \mathbb{J}_{i}} v_{i j}^{2}\left\|\tau_{Z_{i j}} \Lambda_{i} \pi_{W_{i}}(f)\right\|^{2} \leq(\sqrt{\delta}+\sqrt{\beta})^{2}\|f\|^{2}, \quad \forall f \in H .
$$

Similarly we have

$$
\sum_{i \in \mathbb{I}} \sum_{j \in \mathbb{J}_{i}} v_{i j}^{2}\left\|\tau_{Z_{i j}} \Lambda_{i} \pi_{W_{i}}(f)\right\|^{2} \geq(\sqrt{\delta}-\sqrt{\alpha})^{2}\|f\|^{2}, \quad \forall f \in H .
$$

We remark that there also exist other forms of perturbation about the families of operators $\left\{\Lambda_{i}\right\}_{i \in \mathbb{I}}$ and subspaces $\left\{W_{i}\right\}_{i \in \mathbb{I}}$ of RFFs. These results are analogous to Theorem 5.1 and Theorem 5.2 with the proofs following similar lines, therefore we omit them. 


\section{Acknowledgements}

The authors wish to thank the anonymous reviewers for their valuable comments and suggestions that have improved the presentation of this paper.

Funding

This work is supported by National Natural Science Foundation of P.R. China (No. 11671201).

Availability of data and materials

Data sharing not applicable to this article as no datasets were generated or analysed during the current study.

\section{Ethics approval and consent to participate}

Not applicable.

\section{Competing interests}

The authors declare that they have no competing interests.

\section{Consent for publication}

Not applicable.

\section{Authors' contributions}

All authors contributed equally to the writing of the present article. They also read and approved the final manuscript.

\section{Publisher's Note}

Springer Nature remains neutral with regard to jurisdictional claims in published maps and institutional affiliations.

Received: 30 April 2019 Accepted: 14 August 2019 Published online: 27 August 2019

\section{References}

1. Asgari, M.S., Khosravi, A.: Frames and bases of subspaces in Hilbert spaces. J. Math. Anal. Appl. 308(2), 541-553 (2005)

2. Cazassa, P.G.: The art of frame theory. Taiwan. J. Math. 4(2), 129-202 (2000)

3. Cazassa, P.G., Christensen, O.: Perturbation of operators and applications to frame theory. J. Fourier Anal. Appl. 3(5), 543-557 (1997)

4. Cazassa, P.G., Kutyniok, G.: Frames of subspaces. Contemp. Math. 345, 87-113 (2003)

5. Cazassa, P.G., Kutyniok, G., Li, S.: Fusion frames and distributed processing. Appl. Comput. Harmon. Anal. 25(1), 114-132 (2008)

6. Christensen, O.: An Introduction to Frames and Riesz Bases. Birkhäuser, Boston (2003)

7. Christensen, O., Heil, C.: Perturbations of Banach frames and atomic decompositions. Math. Nachr. 185(1), 33-47 (1997)

8. Diestel, J.: Sequences and Series in Banach Spaces. Springer, Berlin (1984)

9. Duffina, R.J., Schaeffer, A.C.: A class of nonharmonic Fourier series. Trans. Am. Math. Soc. 72(2), 341-366 (1952)

10. Găvruta, P:: On the duality of fusion frames. J. Math. Anal. Appl. 333(2), 871-879 (2007)

11. Han, D., Larson, D.R.: Frames, bases and group representations. Mem. Am. Math. Soc. 147, 697 (2000)

12. Hausl, C., Hagenauer, J.: Relay communication with hierarchical modulation. IEEE Commun. Lett. 11(1), 64-66 (2007)

13. Heineken, S.B., Morillas, P.M.: Properties of finite dual fusion frames. Linear Algebra Appl. 453, 1-27 (2014)

14. Heineken, S.B., Morillas, P.M., Benavente, A.M., Zakowicz, M.I.: Dual fusion frames. Arch. Math. 103(4), 355-365 (2014)

15. Kolster, F.A.: Generation and utilization of ultra-short waves in radio communication. Proc. IRE 22(12), 1335-1353 (1934)

16. Masse, D.: Radio-frequency and microwave communication circuits analysis and design, 2nd edn. (2001)

17. Noneman, E.E.: Tracking and data relay satellite system. In: Space Tracking and Data Systems. Proceedings of the Symposium, pp. 77-88 (1979)

18. Nosratinia, A., Hunter, T.E., Hedayat, A.: Cooperative communication in wireless networks. IEEE Commun. Mag. 42(10), 74-80 (2004)

19. Ruiz, M.A., Stojanoff, D.: Some properties of frames of subspaces obtained by operator theory methods. J. Math. Anal. Appl. 343(1), 366-378 (2008)

20. Slimane, S.B., Osseiran, A.: Relay communication with delay diversity for future communication systems. In: Vehicular Technology Conference IEEE. (2006)

21. Sun, W.: G-frames and g-Riesz bases. J. Math. Anal. Appl. 322(1), 437-452 (2006)

22. Sun, W.: Stability of G-frames. J. Math. Anal. Appl. 326(2), 858-868 (2007)

23. Tao, B., Campbell, J., Griffiths, G.: Distributed sensor relay system for near real time observation, control and data management on a scientific research ship. In: Benlamri, R. (ed.) Networked Digital Technologies. NDT. Communications in Computer and Information Science, vol. 293. Springer, Berlin (2012)

24. Young, R.M.: An Introduction to Nonharmonic Fourier Series. Academic Press, New York (1980) 